Pacific

Journal of

Mathematics

ENDO-WILD REPRESENTATION TYPE AND GENERIC REPRESENTATIONS OF FINITE POSETS

DAVID M. ARNOLD AND DANIEL Simson 


\title{
ENDO-WILD REPRESENTATION TYPE AND GENERIC REPRESENTATIONS OF FINITE POSETS
}

\author{
DAVID M. ARNOLD AND DANIEL SIMSON
}

Dedicated to the memory of Sheila Brenner

\begin{abstract}
The category of finite-dimensional representations of a finite poset over an arbitrary field $k$ is shown to have $k$-wild representation type if and only if it has $\boldsymbol{k}$-endo-wild representation type. Included are some characterizations of representation type in terms of existence conditions on infinitedimensional generic representations and their endomorphism rings.
\end{abstract}

\section{Introduction}

Let $k$ be an arbitrary field and $(S, \leq)$ a finite partially ordered set (poset) with a partial order relation $\leq$. Throughout we write simply $S$ instead of $(S, \leq)$, and we assume that $S$ has a unique maximal element, denoted by $\infty$. We are interested in the category $\operatorname{fpr}(S, k)$ of $S$-filtered finite-dimensional $k$-linear representations of $S$ with objects $U=\left(U_{s} ; s \in S\right)$, where each $U_{s}$ is a finite-dimensional $k$-vector space and $U_{s} \subseteq U_{t} \subseteq U_{\infty}$ if $s \leq t$ in $S$. A morphism from $U=\left(U_{s} ; s \in S\right)$ to $V=\left(V_{s} ; s \in S\right)$ is a $k$-linear map $f: U_{\infty} \longrightarrow V_{\infty}$ such that $f\left(U_{s}\right) \subseteq V_{s}$ for each $s \in S$.

Notice that $\operatorname{fpr}(S, k)$ is an additive Krull-Schmidt $k$-category, and it is the category $S^{\prime}$-sp of $S^{\prime}$-spaces over $k$ studied in [Simson 1992], where $S^{\prime}=S \backslash\{\infty\}$ is viewed as a subposet of $S$.

We recall that the category $\operatorname{fpr}(S, k)$ is equivalent to a full subcategory of the category $\bmod k S$ of finitely generated modules over the incidence $k$-algebra $k S$ of the poset $S$ with coefficients in $k$; see [Simson 1992, Chapter 5]. Many properties of $\operatorname{fpr}(S, k)$, such as the existence of almost split sequences, are inherited from $\bmod k S$. On the other hand, $\operatorname{fpr}(S, k)$ has properties not enjoyed by $\bmod k S$, or more generally $\bmod A$ for a finite-dimensional $k$-algebra $A$, without some restrictions. For example, the classical Kleiner-Nazarova characterizations of representation type and a tame/wild dichotomy theorem for $\operatorname{fpr}(S, k)$ are independent of $k$ [Nazarova 1975; Zavadskij and Nazarova 1977; Zavadskij 1987; 1991; Arnold and

Arnold was supported by Nicolaus Copernicus University and the Baylor University Research Committee. Simson was supported by Polish KBN Grant P03A 01521. 
Simson 2002], while the only known tame/wild dichotomy theorem for $\bmod A$, where $A$ is a finite-dimensional $k$-algebra, has the assumption that $k$ is an algebraically closed field [Drozd 1979; Crawley-Boevey 1988].

In this paper, we study two other properties of $\operatorname{fpr}(S, k)$. The first asserts that $k$-wild representation type is equivalent to $k$-endo-wild representation type for $\operatorname{fpr}(S, k)$, where $k$ is an arbitrary field (Theorem 2.4).

We recall from [Han 2002] that $k$-wild representation type need not be equivalent to $k$-endo-wild representation type, for the category $\bmod A$ of finite-dimensional $A$-modules, even under the assumption that $A$ is a finite-dimensional algebra over an algebraically closed field $k$ (see also [Han 2001a; 2001b]). It is shown in [Han 2002, Theorem 2] that for any radical square zero $k$-algebra $A$ of finite dimension, the category $\bmod A$ is $k$-endo-wild if and only if $\bmod A$ is fully $k$-wild, and $\bmod A$ is Corner type $k$-endo-wild if and only if $\bmod A$ is wild. In [Han 2001a; 2001b; 2002], a connection between $k$-endo-wildness and controlled $k$-wildness for a $k$ algebra is also discussed; see also [Ringel 2002; Ringel and Schmidmeier 2004].

The second property asserts that representation type for $\operatorname{fpr}(S, k)$ can be characterized by existence conditions on generic representations and their endomorphism rings (Theorems 3.4 and 3.8). Similar existence conditions on generic modules for $\bmod A$, where $A$ is a finite-dimensional $k$-algebra over an algebraically closed field $k$, are proved in [Crawley-Boevey 1991] using the theory of bocses. Since some of the reduction algorithms in the theory of bocses are not well developed, unless $k$ is an algebraically closed field, our arguments rely on the special nature of $\operatorname{fpr}(S, k)$ for an arbitrary field $k$.

As demonstrated in [Arnold 2000; Arnold and Simson 2002; Simson 1992], properties of the categories $\mathrm{fspr}(S, R)$ for a commutative uniserial ring $R$ and $\operatorname{fpr}(S, k)$ for an arbitrary field $\mathrm{k}$ have immediate application to categories of abelian groups and lattices over orders. In particular, Theorems 2.4, 3.4 and 3.8 answer some open questions stated in [Arnold 2000] for some quasi-homomorphism categories of torsion-free abelian groups of finite rank. See also [Arnold et al. 1993; Dugas and Rangaswamy 2002; Mader 2000; Nongxa and Vinsonhaler 1996; Richman and Walker 1999; Schmidmeier 2004].

There is a classical theorem in abelian group theory due to A. L. S. Corner [1963]: If $R$ is a ring whose additive group is a reduced torsion-free group of finite rank, then there is a torsion-free abelian group $G$ of finite rank with End $G \cong R$. The resulting diversity of endomorphism rings of torsion-free abelian groups of finite rank allows for the construction of examples of pathological direct sum decompositions [Fuchs 1973]. Since Corner's theorem appeared, a diversity of endomorphism rings has served as a de-facto deterrent to a search for complete sets of invariants of classes of torsion-free abelian groups of finite rank. A consequence of Theorem 2.4, together with a category equivalence of M. C. R. Butler [1968], is that, for the 
quasi-isomorphism category of Butler groups with typesets in a fixed finite lattice of types, $k$-endo-wild representation type is equivalent to $k$-wild representation type. In this case, the imprecise notion that a diversity of endomorphism rings is a deterrent to classification of classes of torsion-free abelian groups of finite rank is made precise.

Throughout this paper we use the standard terminology and notation. In particular, given a ring $R$, we denote by $J(R)$ the Jacobson radical of $R$, by $\operatorname{Mod} R$ the category of all right $R$-modules and by $\bmod R$ the full subcategory of $\operatorname{Mod} R$ formed by finitely generated modules.

\section{2. $k$-wild and $k$-endo-wild representation type}

Let $k$ be a field and $S$ a finite poset with a unique maximal element. Following [Drozd 1979; Simson 1992, Section 14.2; 1993; 2003], we define a full exact subcategory $\mathscr{C}$ of $\operatorname{fpr}(S, k)$ to have $k$-wild representation type if there is an exact $k$-linear functor

$$
T: \text { modf } k\left\langle t_{1}, t_{2}\right\rangle \longrightarrow \mathscr{C}
$$

that preserves isomorphism classes (that is, $T(X) \cong T(Y)$ implies $X \cong Y$ ) and preserves indecomposables, where $k\left\langle t_{1}, t_{2}\right\rangle$ is the polynomial $k$-algebra in two noncommuting variables $t_{1}$ and $t_{2}$ and modf $k\left\langle t_{1}, t_{2}\right\rangle$ is the category of finitely generated right $k\left\langle t_{1}, t_{2}\right\rangle$-modules with finite $k$-dimension. If, in addition, the functor $T$ is full, then $\mathscr{b}$ has fully $k$-wild representation type.

It follows from [Simson 1993] and Corollary 2.3 below that $\mathscr{C}$ has fully $k$-wild representation type if and only if it has strictly $k$-wild representation type in the sense of [Crawley-Boevey 1992]; see also below.

Following [Crawley-Boevey 1992], [Ringel 1976] and [Simson 2003, Remark 2.5], we need to distinguish between $k$-wildness and wildness, because the two notions do not coincide for the category $\bmod R$. We recall that the category $\bmod R$ of finite-dimensional right modules over a finite dimensional $k$-algebra $R$ is defined as wild if there exist a finite field extension $k^{\prime}$ of $k$ and a faithful exact additive functor $T^{\prime}: \operatorname{modf} k^{\prime}\left\langle t_{1}, t_{2}\right\rangle \longrightarrow \bmod R$ that preserves isomorphism classes and preserves indecomposables; moreover, $\bmod R$ is strictly wild if there is such a $T^{\prime}$ that is fully faithful. If $k$ is algebraically closed, $\bmod R$ is $k$-wild if and only if it is wild, and it is fully $k$-wild if and only if it is strictly wild.

Following the definition of fully wild representation type and [Arnold 2000], [Simson 2002, Definition 5.1] and [Simson 2003, Definition 2.6], we introduce the following two useful notions for an arbitrary additive $k$-category.

Definition 2.1. Let $k$ be a field. An additive $k$-category $\mathscr{b}$ is defined to have $k$ endo-wild representation type if for each finite-dimensional $k$-algebra $A$ there is an object $U$ of $\mathscr{C}$ and a $k$-algebra isomorphism End $U \cong A$. 
According to [Han 2002, Theorem 2], for the category $\mathscr{C}=\bmod A$ of $\bmod -$ ules over a radical square zero $k$-algebra $A$ of finite dimension, then $k$-endo-wild representation type and fully $k$-wild representation type coincide.

The following simple lemma is very useful (see [Simson 2003]).

Lemma 2.2. Let $k$ be a field and let $\mathscr{b}$ be a full exact $k$-subcategory of $\operatorname{fpr}(S, k)$.

(a) The category $\mathscr{C}$ has fully $k$-wild representation type if and only if, for each finite-dimensional $k$-algebra $A$, there is a fully faithful exact $k$-linear functor $H: \bmod A \longrightarrow \mathscr{C}$.

(b) If, for each finite-dimensional k-algebra A, there exists a fully faithful $k$-linear functor $H: \bmod A \longrightarrow \mathscr{C}$, then, for each finite-dimensional $k$-algebra $A$, there exists such a functor $H$ that is exact.

(c) If, for each finite-dimensional k-algebra A, there exists a fully faithful $k$-linear functor $H: \bmod A \longrightarrow \mathscr{C}$, then $\mathscr{C}$ has $k$-endo-wild representation type.

Proof. (a) Sufficiency is easy, because for the $k$-algebra

$$
A=\left(\begin{array}{ll}
k & k^{3} \\
0 & k
\end{array}\right)
$$

of $k$-dimension five there exists a fully faithful exact $k$-linear functor

$$
\operatorname{modf} k\left\langle t_{1}, t_{2}\right\rangle \longrightarrow \bmod A
$$

(see [Simson 1992, pp. 286-287]).

Necessity is a consequence of [Brenner 1974a] and [Simson 1993, Theorem 2.9], as follows. Suppose that $\mathscr{C}$ has fully $k$-wild representation type and $A$ is a finite-dimensional $k$-algebra. The theorem just cited says that there exists a fully faithful exact $k$-linear functor $T: \operatorname{modf} k\left\langle t_{1}, t_{2}\right\rangle \longrightarrow \mathscr{C}$. On the other hand, by a well known result from Sheila Brenner [1974a], there is a fully faithful exact $k$ linear functor $F: \bmod A \longrightarrow \operatorname{modf} k\left\langle t_{1}, t_{2}\right\rangle$. It follows that the composite $k$-linear functor $H=T \circ F: \bmod A \longrightarrow \mathscr{C}$ is fully faithful and exact.

(b) The proof of is analogous to that of (a).

(c) Assume that $A$ is a finite-dimensional $k$-algebra. Then there exists a fully faithful $k$-linear functor $H: \bmod A \longrightarrow \mathscr{C}$. It follows that $U=H(A)$ is an object of $\mathscr{C} \subseteq \operatorname{fpr}(S, k)$ such that End $U \cong A$.

The proof of Lemma 2.2, together with [Simson 1993, Theorem 2.9], yields:

Corollary 2.3. Let $k$ be a field. A full exact $k$-subcategory $\mathscr{b}$ of $\operatorname{fpr}(S, k)$ has fully $k$-wild representation type if and only if there exists a fully faithful exact $k$-linear functor $H:$ modf $k\left\langle t_{1}, t_{2}\right\rangle \longrightarrow \mathscr{C}$. In this case $H$ has the form $H=$ 


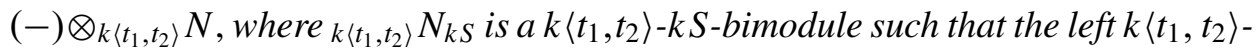
module $k\left\langle t_{1}, t_{2}\right\rangle N$ is free of finite rank.

The arguments applied above show that if $R$ is a fully $k$-wild $k$-algebra, that is, $\bmod R$ is of fully $k$-wild representation type, then the category $\bmod R$ is $k$-endowild. Since not every local wild algebra over an algebraically closed field $k$ is fully wild then, according to [Han 2002, Theorem 2], there exists a $k$-algebra $R$ such that $\bmod R$ is $k$-wild, but it is not $k$-endo-wild. The problem of determining all finite-dimensional $k$-algebras $R$ for which $\bmod R$ has fully $k$-wild representation type if and only if $\bmod R$ has $k$-endo-wild representation type remains unsolved (see [Simson 2002, Problem 5.2]).

Let $U=\left(U_{s} ; s \in S\right)$ be an object of $\operatorname{fpr}(S, k)$. The coordinate vector of $U$ is defined in [Simson 1992] to be the integral vector

$$
\operatorname{cdn} U=\left(u_{s}\right)_{s \in S} \in \mathbb{Z}^{S},
$$

where $u_{s}=\operatorname{dim}_{k}\left(U_{s} / \sum_{t<s} U_{s}\right)$ if $s \in S \backslash\{\infty\}$ and $u_{\infty}=\operatorname{dim}_{k}\left(U_{\infty}\right)$. We set

$$
|\operatorname{cdn} U|=\sum_{s \in S} u_{s}
$$

The representation $U$ is defined to be sincere if $U$ is indecomposable and $u_{s} \neq 0$ for each $s \in S \backslash\{\infty\}$. The poset $S$ is sincere if there is an indecomposable sincere object of $\operatorname{fpr}(S, k)$.

We recall from [Simson 1992, Proposition 5.14] that for any indecomposable object $U$ of $\operatorname{fpr}(S, k)$, with $\operatorname{cdn} U=\left(u_{s}\right)_{s \in S}$, the subposet

$$
S_{U}=\left\{s \in S ; \quad u_{s} \neq 0\right\}
$$

of $S$, called the coordinate support of $U$, is sincere, the restriction $V$ of $U$ to $S_{U}$ is an indecomposable sincere object of $\operatorname{fpr}\left(S_{U}, k\right)$, and there exists a fully faithful $k$-linear embedding $i: \mathrm{fpr}\left(S_{U}, k\right) \longrightarrow \mathrm{fpr}(S, k)$ such that $U \cong i(V)$. In particular, there is a $k$-algebra isomorphism End $U \cong$ End $V$.

Now we show that, for the category $\operatorname{fpr}(S, k)$, the $k$-wild representation and $k$-endo-wild representation type coincide.

Theorem 2.4. Assume that $k$ is an arbitrary field and $S$ is a finite poset with a unique maximal element. The following three statements are equivalent.

(a) The category $\operatorname{fpr}(S, k)$ has $k$-wild representation type.

(b) The category $\operatorname{fpr}(S, k)$ has $k$-endo-wild representation type.

(c) There exists a fully faithful $k$-linear functor $T: \operatorname{modf} k\left\langle t_{1}, t_{2}\right\rangle \longrightarrow \operatorname{fpr}(S, k)$.

(d) The category $\operatorname{fpr}(S, k)$ has fully $k$-wild representation type.

Proof. (d) $\Rightarrow$ (c) is obvious, and (c) $\Rightarrow$ (b) follows from Lemma 2.2(c). 
(a) $\Rightarrow$ (d): Assume that the category $\operatorname{fpr}(S, k)$ has $k$-wild representation type. The poset $S$ contains a subposet $\mathcal{N}$ isomorphic to one of the six hypercritical posets of [Nazarova 1975]:

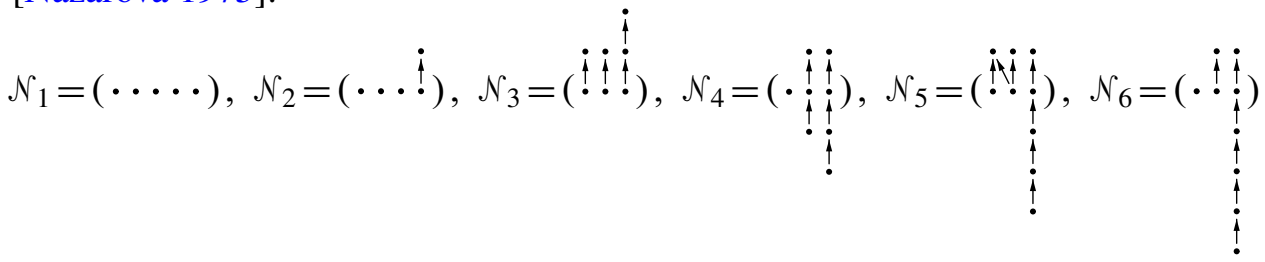

(see also [Simson 1992, Theorem 15.3]). There exists a pair of fully faithful exact $k$-linear functors

$$
\operatorname{modf} k\left\langle t_{1}, t_{2}\right\rangle \stackrel{\widehat{F}_{\mathcal{N}}}{\longrightarrow} \operatorname{prin} k \mathcal{N}^{\infty} \stackrel{\widehat{T}_{\mathcal{N}}}{\longrightarrow} \operatorname{prin} k S,
$$

where, for a poset $T$, prin $k T$ denotes the category of prinjective right $k T$-modules and $T^{\infty}=T \cup\{\infty\}$, with $t<\infty$, for all $t \in T$. The functor $\widehat{F}_{\mathcal{N}}$ is constructed in [Simson 1992, pp. 310-312] (and corrected in Theorem A.1 of the Appendix), whereas $\widehat{T}_{\mathcal{N}}$ is the subposet induction functor ([Simson 1992, (11.85)]; see also [Kasjan and Simson 1995]). This shows that the subcategory prin $k S$ of $\bmod k S$ has fully $k$-wild representation type. By applying [Kasjan 2003, Proposition 5.4] to the poset $S^{\prime}=S \backslash\{\infty\}$ and to the adjustment functor $\Theta: \operatorname{prin} k S \longrightarrow S^{\prime}$-sp $\cong \bmod _{\mathrm{sp}} k S$ defined in [Simson 1992, 11.32-34], we conclude that the category $\operatorname{fpr}(S, k) \cong$ $\bmod _{\text {sp }} k S$ has fully $k$-wild representation type, and (d) follows.

(b) $\Rightarrow$ (a): Assume that $\operatorname{fpr}(S, k)$ does not have $k$-wild representation type. As a consequence of Nazarova's theorem [1975] (see also [Zavadskij and Nazarova 1977; Simson 1992, Theorem 15.3]), the poset $S$ does not contain, as a subposet, any of the six hypercritical posets $\mathcal{N}_{1}, \mathcal{N}_{2}, \mathcal{N}_{3}, \mathcal{N}_{4}, \mathcal{N}_{5}, \mathcal{N}_{6}$ of Nazarova [Nazarova 1975]. There are several cases to be considered in order to show that $\operatorname{fpr}(S, k)$ does not have $k$-endo-wild representation type. The analysis of these cases will occupy us until the end of this section.

Case I. $S=(\cdots) \cup\{\infty\}$.

By [Brenner 1974b], for each indecomposable object $U$ of $\operatorname{fpr}(S, k)$, the algebra End $U$ is isomorphic to a quotient algebra of the polynomial algebra $k[x]$. In particular, either $U$ is preprojective or preinjective with End $U \cong k$ or else $U$ is regular with End $U \cong k[x] /\left(g(x)^{e}\right)$, for some irreducible polynomial $g(x)$ (see also [Simson 1992, Section 15.6; Zavadskij 1987; 1991]). This shows that $\operatorname{fpr}(S, k)$ does not have $k$-endo-wild representation type, because the matrix $k$-subalgebra

$$
A=\left\{\left(\begin{array}{lll}
a & b & d \\
0 & a & c \\
0 & 0 & a
\end{array}\right) ; a, b, c, d \in k\right\}
$$


of $\mathbb{M}_{3}(K)$ of dimension four has no nontrivial idempotents and it is not isomorphic to an algebra $k[x] /\left(g(x)^{e}\right)$, for some irreducible polynomial $g(x)$.

Case II. The poset $S$ does not contain, as a subposet, any of the posets $\mathcal{N}_{1}, \mathcal{N}_{2}, \mathcal{N}_{3}$, $\mathcal{N}_{4}, \mathcal{N}_{5}, \mathcal{N}_{6}$, or

$$
\mathcal{N} \mathscr{L}=(\stackrel{i \times i}{\cdot} \cdot \cdot) \text {. }
$$

It is sufficient to prove that the algebra End $U$ is commutative, for each sincere indecomposable object $U$ of $\operatorname{fpr}(S, k)$, recalling that each indecomposable object in $\operatorname{fpr}(S, k)$ may be identified with a sincere indecomposable object in $\operatorname{fpr}\left(S_{U}, k\right)$, for some subposet $S_{U}$ of $S$. In this case, $\operatorname{fpr}(S, k)$ does not have $k$-endo-wild representation type since, for example, the $k$-algebra $A$ of dimension four defined in Case I is noncommutative and has no nontrivial idempotents.

The proof that End $U$ is commutative, for each sincere indecomposable object $U$ of $\operatorname{fpr}(S, k)$, is an induction on

$$
p(U)=(|\operatorname{cdn} U|, v(U)) \in \mathbb{N} \times \mathbb{N}^{o p},
$$

where $v(U)$ is the cardinality of the set $\left\{(s, t) ; s<t\right.$ in $\left.S, u_{s} \neq 0, u_{t} \neq 0\right\}, \mathbb{N} \times \mathbb{N}^{o p}$ is ordered lexicographically and $\operatorname{cdn} U=\left(u_{s}\right)_{s \in S}$, see [Simson 1992, proof of Theorem 15.54].

If $S=(\cdots) \cup\{\infty\}$, then End $U$ is commutative, by Case I.

Now assume that $S \neq(\cdots) \cup\{\infty\}$ and $S$ does not contain any of the posets $\mathcal{N}_{1}, \mathcal{N}_{2}, \mathcal{N}_{3}, \mathcal{N}_{4}, \mathcal{N}_{5}, \mathcal{N}_{6}, \mathcal{N}_{\mathscr{L}}$, as a subposet. In view of [Simson 1992, Theorem 15.30], there exists an irreducible suitable pair $(a, b)$ of elements of $S$. Let $\delta_{(a, b)}$ : $\operatorname{fpr}(S, k) \longrightarrow \operatorname{fpr}\left(\delta_{(a, b)} S, k\right)$ denote the Zavadskij derivative (see [Zavadskij 1977; 1991; Simson 1992, Definition 9.2]) with respect to $(a, b)$.

Define $S_{a<b}$ to be the poset obtained from $S$ by adding the relation $a<b$ and let $u: \operatorname{fpr}\left(S_{a<b}, k\right) \longrightarrow \operatorname{fpr}(S, k)$ be the natural fully faithful embedding. Given a sincere indecomposable object $U$ of $\operatorname{fpr}(S, k)$, then either $U^{\prime}=\delta_{(a, b)}(U)$ is a sincere indecomposable object of $\operatorname{fpr}\left(\delta_{(a, b)} S, k\right)$, with $p\left(U^{\prime}\right)<p(U)$, or else $U_{a} \subseteq$ $U_{b}$ and $U=u\left(U^{\prime}\right)$, for some sincere indecomposable object $U^{\prime}$ of $\operatorname{fpr}\left(S_{a<b}, k\right)$, with $p\left(U^{\prime}\right)<p(U)$, [Simson 1992, Lemma 15.52]. Since $\delta_{(a, b)} S$ does not contain any of the posets $\mathcal{N}_{1}, \mathcal{N}_{2}, \mathcal{N}_{3}, \mathcal{N}_{4}, \mathcal{N}_{5}, \mathcal{N}_{6}, \mathcal{N} \mathscr{E}$, as a subposet, [Simson 1992, Proposition 15.27 and Theorem 15.30], then the algebra End $U^{\prime}$ is commutative, by induction on $p(U)$.

If $U=u\left(U^{\prime}\right)$, then End $U \cong$ End $U^{\prime}$ is commutative, since $u$ is a fully faithful functor.

Now suppose that $U^{\prime}=\delta_{(a, b)}(U)$. If $U$ is isomorphic to $P_{a}$ (an indecomposable projective based on $a$, with one-dimensional peak space) or $P \cong P_{a, c_{i}}=P_{a}+P_{c_{i}}$ (an indecomposable subobject of the injective envelope $E\left(P_{\infty}\right)$ of $P_{\infty}$ generated by $P_{a}$ and $P_{c_{i}}$, see [Simson 1992, Chapter 5]), then End $U \cong k$. 
Next, assume that $U$ is sincere and indecomposable, but not isomorphic to $P_{a}$ or any $P_{a, c_{i}}$. By applying Lemma 9.13 and Corollary 9.18 of [Simson 1992], we show that End $U^{\prime} \cong$ End $W$.

To see this, we note that, by Proposition 9.16 of [Simson 1992], the functor $\delta_{(a, b)}^{\prime}$ induces a $k$-algebra isomorphism $\phi:$ End $U \rightarrow($ End $W) / \mathscr{A}_{W}$, where

$$
\mathscr{A}_{W}=\left\{f \in \text { End } W: f \text { factors through } P_{a}^{t} \text { for some } t\right\} .
$$

Now $\mathscr{A}_{W} \subseteq \operatorname{Hom}\left(W, W_{(\geq a)}\right)$, where $W_{(\geq a)}=\left(U_{s}: s \in \delta_{(a, b)} S\right)$, with $U_{s}=W_{s}$, if $s \geq a$, and $U_{s}=0$, if $s \ngtr a$.

Assume $\mathscr{A}_{W} \neq 0$. Then $W_{a} \neq 0$, because $W_{a}=0$ would imply $\operatorname{Hom}\left(P_{a}, W\right) \cong$ $W_{a}=0$. Since $\mathscr{A}_{W} \neq 0$ and $W_{a} \neq 0$, there is a morphism $W \rightarrow W_{(\geq a)}$ with image isomorphic to $P_{a}$. This is a contradiction to the assumption that $W$ is indecomposable and not isomorphic to $P_{a}$ and the fact that $P_{a}$ is projective. Consequently, $\mathscr{A}_{W}=0$ and End $U \cong$ End $W \cong$ End $U^{\prime}$ is commutative, as desired.

Case III. $S$ does not contain any one of $\mathcal{N}_{1}, \mathcal{N}_{2}, \mathcal{N}_{3}, \mathcal{N}_{4}, \mathcal{N}_{5}, \mathcal{N}_{6}$, but does contain $\mathcal{N} \mathscr{L}$ as a subposet.

As noted in Case II, it is sufficient to assume that $S$ is a sincere poset. Then $S$ is a union of two garlands [Simson 1992, Proposition 15.61], where a garland is a poset of width 2 that does not contain the poset $(1,2)$ as a subposet.

Define $\varphi_{m, n}$ to be the disjoint union of two garlands $\varphi_{m}$ and $\varphi_{n}$, where $\varphi_{m}$ is a garland with exactly $m$ pairs of incomparable elements, that is, it has the form

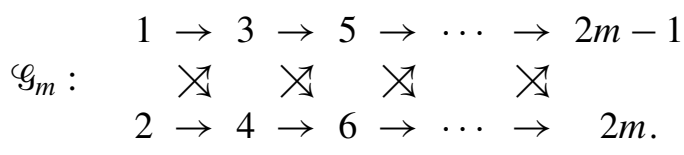

As in [Simson 1992, Corollary 15.63], $\operatorname{fpr}(S, k)$ does not have $k$-endo-wild representation type if $\operatorname{fpr}\left(\varphi_{m, n}^{\infty}, k\right)$ does not have $k$-endo-wild representation type.

It remains to prove that $\operatorname{fpr}\left(\mathscr{G}_{m, n}^{\infty}, k\right)$ does not have $k$-endo-wild representation type. The category $\operatorname{fpr}\left(\mathscr{G}_{m, n}^{\infty}, k\right)$ is equivalent to the category $\operatorname{rep}_{k}\left(\mathscr{C}_{m, n}\right)$ of $k$-linear representations of a nonsingular separably reducible clan $\mathscr{C}_{m, n}$ associated to $\mathscr{G}_{m, n}$ in [Simson 1992, Lemma 15.67]. Indecomposable objects $W$ of $\operatorname{rep}_{k}\left(\mathscr{C}_{m, n}\right)$ are classified in terms of $k$-linear functors $S_{w}: \operatorname{modf} A_{w} \longrightarrow \operatorname{rep}_{k}\left(\mathscr{C}_{m, n}\right)$ indexed by words $w$ as follows, see [Crawley-Boevey 1989]:

(a) $W$ is an asymmetric string isomorphic to $S_{w}(M)$ for some indecomposable $M$ in modf $A_{w}$, where $A_{w}=k ;$

(b) $W$ is a symmetric string isomorphic to $S_{w}(M)$ for some indecomposable module $M$ in modf $A_{w}$, where $A_{w}=k[x] /(q(x))$ and $q(x)$ is a quadratic polynomial with nonzero constant term and distinct roots in $k$; 
(c) $W$ is an asymmetric band isomorphic to $S_{w}(M)$ for some indecomposable $M$ in modf $A_{w}$, where $A_{w}=k\left[x, x^{-1}\right]$;

(d) $W$ is a symmetric band isomorphic to $S_{w}(M)$ for some indecomposable $M$ in modf $A_{w}$, where $A_{w}=k\left\langle t_{1}, t_{2}\right\rangle /\left(p\left(t_{1}\right), q\left(t_{2}\right)\right)$ and $p\left(t_{1}\right)$ and $q\left(t_{2}\right)$ are quadratic polynomials with nonzero constant terms and distinct roots in $k$.

Moreover, there are $k$-linear functors $F_{w}: \operatorname{rep}_{k}\left(\mathscr{C}_{m, n}\right) \longrightarrow \operatorname{modf} A_{w}$, with $F_{w} \circ S_{w}$ naturally equivalent to the identity functor on modf $A_{w}$.

This is proved in [Crawley-Boevey 1989], when the field $k$ has at least three elements, and in [Deng 2000], when the field $k$ is arbitrary.

It now follows that, for each indecomposable object $W$ of $\operatorname{rep}_{k}\left(\mathscr{C}_{m, n}\right)$, there is some $A_{w}$, an indecomposable module $M$ in modf $A_{w}$, and an ideal $I_{w}$ of End $W$ with $($ End $W) / I_{w} \cong \operatorname{End}_{A_{w}} M$ and $F_{w *}\left(I_{w}\right)=0$, where the map $F_{w}^{*}:$ End $W \longrightarrow$ $\operatorname{End}_{A_{w}} M$ is induced by the functor $F_{w}$. Because $W$ is indecomposable, End $W$ is a local ring so that $I_{w} \subseteq J$ (End $W$ ). Hence, there is a $k$-algebra isomorphism

$$
\text { End } W / J(\text { End } W) \cong \operatorname{End}_{A_{w}} M / J\left(\operatorname{End}_{A_{w}} M\right) \text {. }
$$

In each case, $\operatorname{End}_{A_{w}} M$ is isomorphic to a quotient algebra of $k[x]$. This is because indecomposable modules in modf $A_{w}$ are cyclic $k[x]$-modules in the case of types (a)-(c), as we can see by observing that $k\left[x, x^{-1}\right]$ is a localization of $k[x]$.

Next, we show that (d) is a consequence of Case I.

Let $S=(\cdots) \cup\{\infty\}$ and $A_{w}=k\left\langle t_{1}, t_{2}\right\rangle /\left(p\left(t_{1}\right), q\left(t_{2}\right)\right)$, where $p\left(t_{1}\right)$ and $q\left(t_{2}\right)$ are quadratic polynomials with nonzero constant terms and distinct roots in $k$. Assume that $p\left(t_{1}\right)=\left(t_{1}-\lambda_{1}\right)\left(t_{1}-\lambda_{2}\right)$ and $q\left(t_{2}\right)=\left(t_{2}-\lambda_{3}\right)\left(t_{2}-\lambda_{4}\right)$, where $\lambda_{1}, \lambda_{2}, \lambda_{3}, \lambda_{4}$ are nonzero distinct elements of $k$. Define a functor $F$ : modf $A_{w} \longrightarrow \operatorname{fpr}(S, k)$ by the formula $F(M)=\left(M, M_{1}, M_{2}, M_{3}, M_{4}\right)$, where $M_{j}$ is the eigenspace of $\lambda_{j}$. Since $F$ induces the $k$-algebra isomorphism $F_{*}$ : $\operatorname{End}_{A_{w}} M \longrightarrow$ End $F(M)$, we conclude as in Case I that if $M$ is an indecomposable module in modf $A_{w}$, then $\operatorname{End}_{A_{w}} M$ is isomorphic to a quotient $k$-algebra of the polynomial algebra $k[x]$.

In summary, End $W / J($ End $W$ ) is a finite-dimensional field extension of $k$, for each indecomposable object $W$ of $\operatorname{rep}_{k}\left(\mathscr{C}_{m, n}\right)$.

Case III (i). There is a noncommutative finite dimensional division $k$-algebra D.

Since $D$ is noncommutative, there is no indecomposable object $W$ of $\operatorname{rep}_{k}\left(\mathscr{C}_{m, n}\right)$ with End $W / J($ End $W) \cong D$. Therefore, $\operatorname{fpr}\left(\mathscr{G}_{m, n}^{\infty}, k\right)$, and consequently $\operatorname{fpr}(S, k)$, does not have $k$-endo-wild representation type.

Case III(ii). Every finite-dimensional division k-algebra is a field (e.g., $k$ is finite or algebraically closed).

It is sufficient to prove that the category $\operatorname{rep}_{k}\left(\mathscr{C}_{m, n}\right)$ is not $k$-endo-wild. 
- Assume that $W$ is a band representation in $\operatorname{rep}_{k}\left(\mathscr{C}_{m, n}\right)$ (types (c) or (d) above).

The functor $S_{w}: \bmod A_{w} \rightarrow \operatorname{rep}_{k}\left(\mathscr{C}_{m, n}\right)$ preserves irreducible morphisms, hence Auslander-Reiten sequences, in (c) and (d); see [Geiß 1999, Proposition 4]. Since the functor $S_{w}: \bmod A_{w} \rightarrow \operatorname{rep}_{k}\left(\mathscr{C}_{m, n}\right)$ preserves irreducible morphisms, $S_{w}$ induces an isomorphism

$$
\operatorname{Irr}(M, M)=J(M, M) / J^{2}(M, M) \longrightarrow \operatorname{Irr}(W, W)=J(W, W) / J^{2}(W, W),
$$

with notation as in [Simson 1992], where $M$ is a module in $\bmod A_{w}$, with $S_{w}(M)=$ $W$. Since End $M$ and End $W$ are local rings, we have isomorphisms

$$
\operatorname{Irr}(M, M)=J(\text { End } M) / J(\text { End } M)^{2} \text { and } \operatorname{Irr}(W, W)=J(\text { End } W) / J(\text { End } W)^{2} .
$$

For (c) and (d), $J($ End $M) / J(\text { End } M)^{2} \cong k$. Possibility (c) is obvious, and (d) follows as above by reducing to Case I and showing that End $W$ is a factor ring of $k[x]$, for each indecomposable $W$ in $\operatorname{fpr}\left(\varphi_{m, n}^{\infty}, k\right)$.

This shows that $J($ End $W) / J(\text { End } W)^{2} \cong k$ for each band representation $W$ in $\operatorname{rep}_{k}\left(\mathscr{C}_{m, n}\right)$. Because the algebra $A$ defined in Case I is of dimension four, has no nontrivial idempotents and $J(A) / J(A)^{2}$ is noncommutative, then $A$ is not isomorphic to End $W$, for any indecomposable band representation $W$ in $\operatorname{rep}_{k}\left(\mathscr{C}_{m, n}\right)$.

- Now assume instead that $W$ is a string representation in $\operatorname{rep}_{k}\left(\mathscr{C}_{m, n}\right)$.

It is sufficient to prove that End $W$ is not isomorphic to the $k$-algebra $A$ defined in Case I. The problem reduces to the case $m=2, n=1$, where $\mathscr{G}_{m, n}=\mathscr{G}_{2,1}=\mathcal{N} \mathscr{L}$. The clan $\mathscr{C}_{2,1}$ corresponding to the poset $\mathcal{N} \mathscr{Z}$ and the category rep ${ }_{k}\left(\mathscr{C}_{2,1}\right)$ are presented in [Simson 1992, p. 360]. By applying this description and the arguments given [Geiß 1999] and [Krause 1991], one can show, by a detailed technical argument (not included), that the $k$-algebra $A$ defined in Case I is not of the form End $W$, up to isomorphism, where $W$ is a string representation in $\operatorname{rep}_{k}\left(\mathscr{C}_{2,1}\right)$. This finishes the proof of Case III(ii), and hence also of Theorem 2.4.

\section{Generic representations and representation type}

Let $(S, \leq)$ be a finite poset with a unique maximal element $\infty$ and $R$ a unitary ring. Following [Arnold and Simson 2002], we define the category $\operatorname{rep}_{f g}(S, R)$ to be the category of filtered finitely generated $R$-representations of $S$ with objects $U=\left(U_{s} ; s \in S\right)$ such that each $U_{s}$ is a finitely generated left $R$-module and $U_{s} \subseteq U_{t}$ if $s \leq t$ in $S$. A morphism from $U=\left(U_{s} ; s \in S\right)$ to $V=\left(V_{s} ; s \in S\right)$ is an $R$-module homomorphism $f: U_{\infty} \rightarrow V_{\infty}$ such that $f\left(U_{s}\right) \subseteq V_{s}$, for each $s \in S$.

Let $\operatorname{fspr}(S, R)$ denote the full subcategory of filtered subprojective representations of $\operatorname{rep}_{f g}(S, R)$ with objects $U=\left(U_{s} ; s \in S\right)$ such that $U_{\infty}$ is a projective $R$-module. Define $\operatorname{fpr}(S, R)$ to be the full subcategory of $\operatorname{fspr}(S, R)$ with 
$U=\left(U_{s} ; s \in S\right)$ such that $U_{s}$ and $U_{\infty} / U_{s}$ are free $R$-modules, for each $s \in S$. If $k$ is a field, then $\operatorname{fspr}(S, k)=\operatorname{fpr}(S, k)=\operatorname{rep}_{f g}(S, k)$.

For a field $k$, we define $\operatorname{Fpr}(S, k)$ to be the category with objects $U=\left(U_{s} ; s \in S\right)$ such that each $U_{s}$ is a $k$-vector space and $U_{s} \subseteq U_{t}$ if $s \leq t$ in $S$. A morphism from $U=\left(U_{s} ; s \in S\right)$ to $V=\left(V_{s} ; s \in S\right)$ is a $k$-linear transformation $f: U_{\infty} \rightarrow V_{\infty}$ with $f\left(U_{s}\right) \subseteq V_{s}$ for each $s \in S$. The peak $R$-rank of an object $U=\left(U_{s} ; s \in S\right)$ of $\operatorname{Fpr}(S, R)$ is defined to be the $R$-rank of the free $R$-module $U_{\infty}$. The category $\operatorname{Fpr}(S, k)$ is an extension of $\operatorname{fpr}(S, k)$ in that $U_{\infty}$ may be infinite-dimensional.

We write End $U$ for the endomorphism ring of an object $U$ of $\operatorname{Fpr}(S, k)$.

We recall from [Simson 1992, Section 5.1] that the category $\operatorname{Fpr}(S, k)$ may be viewed as a full exact subcategory of the module category Mod $k S$ over the incidence $k$-algebra $k S$ of $S$, because there is a fully faithful $k$-linear exact functor

$$
\rho: \operatorname{Fpr}(S, k) \longrightarrow \operatorname{Mod} k S
$$

which associates to any object $M=\left(M_{s} ; s \in S\right)$ of $\operatorname{Fpr}(S, k)$ the $k$-vector space $\rho(M)=\bigoplus_{s \in S} M_{s}$ equipped with the right multiplication $\cdot: \rho(M) \times k S \rightarrow \rho(M)$ defined by attaching to every element $m=\left(m_{s}\right)_{s \in S} \in \bigoplus_{s \in S} M_{s}=\rho(M)$ and any matrix $\lambda=\left[\lambda_{p q}\right]_{p, q \in S} \in k S \subseteq \mathbb{M}_{S}(k)$ the vector $m \cdot \lambda=\left(m_{s}\right) \cdot\left[\lambda_{p q}\right]=\left(m_{s}^{\prime}\right)_{s \in S} \in$ $\bigoplus_{s \in S} M_{s}=\rho(M)$, where $m_{s}^{\prime}=\sum_{i \leq s} m_{i} \lambda_{i s}$. If $f: M \rightarrow M^{\prime}$ is a morphism in $\operatorname{Fpr}(S, k)$ defined by a $k$-linear map $f: M_{\infty} \rightarrow M_{\infty}^{\prime}$, we define the $k S$-module homomorphism $\rho(f): \rho(M) \rightarrow \rho\left(M^{\prime}\right)$ by the formula $\rho(f)(m)=\left(f\left(m_{s}\right)\right)_{s \in S}$, for every $m=\left(m_{s}\right)_{s \in S} \in \rho(M)$.

The functor $\rho$ allows us to identify the category $\operatorname{Fpr}(S, k)$ with a full exact subcategory of the module category $\operatorname{Mod} k S$. By identifying any object $M=\left(M_{s} ; s \in\right.$ $S)$ of $\operatorname{Fpr}(S, k)$ with the right $k S$-module $\rho(M)$ we can view $M$ as a left module over the endomorphism $k$-algebra End $M \cong \operatorname{End}_{k S} \rho(M)$.

Lemma 3.1. Let $k$ be a field and $S$ a finite poset with a unique maximal element. The category $\operatorname{fpr}(S, k)$ has $k$-wild representation type if and only if there exists an object $N$ of $\operatorname{fpr}\left(S, k\left\langle t_{1}, t_{2}\right\rangle\right)$ such that the $k$-linear functor

$$
-\otimes_{k\left\langle t_{1}, t_{2}\right\rangle} N: \operatorname{Mod} k\left\langle t_{1}, t_{2}\right\rangle \longrightarrow \operatorname{Fpr}(S, k)
$$

is exact, faithful, preserves indecomposables, preserves isomorphism classes and restricts to the functor $-\otimes_{k\left\langle t_{1}, t_{2}\right\rangle} N: \operatorname{modf} k\left\langle t_{1}, t_{2}\right\rangle \longrightarrow \mathrm{fpr}(S, k)$.

Proof. The proof of sufficiency is obvious. To prove necessity, we assume that $\operatorname{fpr}(S, k)$ has $k$-wild representation type, that is, there exists an exact $k$-linear functor

$$
T^{\prime}: \operatorname{modf} k\left\langle t_{1}, t_{2}\right\rangle \longrightarrow \operatorname{fpr}(S, k)
$$


that preserves isomorphism classes and preserves indecomposables. By a well known result of Brenner [1974a], there is a fully faithful exact $k$-linear functor

$$
T^{\prime \prime}: \bmod A \longrightarrow \operatorname{modf} k\left\langle t_{1}, t_{2}\right\rangle, \quad \text { where } A=\left(\begin{array}{ll}
k & k^{3} \\
0 & k
\end{array}\right) .
$$

It follows that the composite $k$-linear functor $T^{\prime} \circ T^{\prime \prime}: \bmod A \longrightarrow \operatorname{fpr}(S, k) \subseteq$ $\operatorname{Mod} k S$ is exact, preserves isomorphism classes and preserves indecomposables. By the Wildness Correction Lemma [Simson 1993, Lemma 2.6] applied to $T^{\prime} \circ T^{\prime \prime}$, there exists a finite-dimensional $A$ - $k S$-bimodule ${ }_{A} M_{k S}^{\prime}$ such that the left $A$-module ${ }_{A} M^{\prime}$ is free of finite rank and the $k$-linear functor

$$
H^{\prime}=-\otimes{ }_{A} M_{k S}^{\prime}: \bmod A \longrightarrow \mathrm{fpr}(S, k) \subseteq \operatorname{Mod} k S
$$

is exact, faithful, preserves isomorphism classes and preserves indecomposables. Moreover, it follows from the proof of [Simson 1993, Lemma 2.6] that the functor $H^{\prime}$ extends to a unique $k$-linear exact faithful functor

$$
H^{\prime}=-\otimes_{A} M_{k S}^{\prime}: \operatorname{Mod} A \longrightarrow \operatorname{Fpr}(S, k) \subseteq \operatorname{Mod} k S,
$$

that preserves isomorphism classes and preserves indecomposables. By [Simson 1992, pp. 286-287] and the arguments used in the proof, there exists a fully faithful exact $k$-linear functor $H^{\prime \prime}: \operatorname{Mod} k\left\langle t_{1}, t_{2}\right\rangle \longrightarrow \operatorname{Mod} A$, which restricts to $H^{\prime \prime}: \operatorname{modf} k\left\langle t_{1}, t_{2}\right\rangle \longrightarrow \bmod A$ and is of the form $H^{\prime \prime}=-\otimes_{k\left\langle t_{1}, t_{2}\right\rangle} M_{A}^{\prime \prime}$, where $k\left\langle t_{1}, t_{2}\right\rangle M_{A}^{\prime \prime}$ is an $k\left\langle t_{1}, t_{2}\right\rangle$-A-bimodule such that the left $k\left\langle t_{1}, t_{2}\right\rangle$-module $M^{\prime \prime}$ is free of finite rank. Since the right $k S$-module ${ }_{A} M_{k S}^{\prime}=A \otimes \otimes_{A} M_{k S}^{\prime}=H^{\prime}(A)$ is an object of $\operatorname{fspr}(S, k)$ of the form $\left({ }_{A} M_{s}^{\prime} ; s \in S\right)$, then the $k\left\langle t_{1}, t_{2}\right\rangle-k S$-bimodule

$$
k\left\langle t_{1}, t_{2}\right\rangle N_{k S}={ }_{k\left\langle t_{1}, t_{2}\right\rangle} M^{\prime \prime} \otimes{ }_{A} M_{k S}^{\prime}
$$

can be identified with the object $\left({ }_{k\left\langle t_{1}, t_{2}\right\rangle} N_{s} ; s \in S\right)$ of the category $\operatorname{fpr}\left(S, k\left\langle t_{1}, t_{2}\right\rangle\right)$, where ${ }_{k\left\langle t_{1}, t_{2}\right\rangle} N_{s}={ }_{k\left\langle t_{1}, t_{2}\right\rangle} M^{\prime \prime} \otimes{ }_{A} M_{s}^{\prime}$ is a free left $k\left\langle t_{1}, t_{2}\right\rangle$-module of finite rank, for each $s \in S$. It follows that the composite $k$-linear functor $H^{\prime} \circ H^{\prime \prime}$ is fully faithful, exact, preserves isomorphism classes, preserves indecomposables, is of the form

$$
-\otimes_{k\left\langle t_{1}, t_{2}\right\rangle} N_{k S}: \operatorname{Mod} k\left\langle t_{1}, t_{2}\right\rangle \longrightarrow \operatorname{Fpr}(S, k)
$$

and restricts to the functor $-\otimes_{k\left\langle t_{1}, t_{2}\right\rangle} N_{k S}: \operatorname{modf} k\left\langle t_{1}, t_{2}\right\rangle \longrightarrow \mathrm{fpr}(S, k)$.

To define generic representations of $S$, we define the endolength of $M$ to be the (composition) length of the left End $U$-module $M$, compare with [CrawleyBoevey 1991]. Moreover, we define the object $M=\left(M_{s} ; s \in S\right)$ of $\operatorname{Fpr}(S, k)$ to be a generic representation of $S$, if $M$ is indecomposable, the $k$-dimension of $M_{\infty}$ is infinite, $M$ has finite endolength, and the $k$-algebra

$$
D_{M}=\text { End } M / J(\text { End } M)
$$


contains, as a $k$-subalgebra, the quotient field $k(x)$ of the polynomial $k$-algebra $k[x]$ in one indeterminate $x$.

By definition, the object $M=\left(M_{s} ; s \in S\right)$ of $\operatorname{Fpr}(S, k)$ is a generic representation of $S$ if and only if $\rho(M)$ is a generic $k S$-module (in the sense of [Crawley-Boevey 1991], that is, $\boldsymbol{\rho}(M)$ is indecomposable of infinite length as a $k S$-module, but is of finite endolength) with the additional property that $k(x) \subseteq D_{M}$.

If the field $k$ is algebraically closed, then the condition that $k(x) \subseteq D_{M}$ is redundant. This is because, by Lemma 3.2, $D_{M}$ is a division $k$-algebra of infinite $k$-dimension (since $M$ has finite $D_{M}$-dimension equal to the endolength of $M$ ). Under the assumption that $k$ is algebraically closed, the division $k$-algebra $D_{M}$ contains an element $x$ transcendental over $k$ and therefore $k(x) \subseteq D_{M}$.

The standard properties of generic modules proved in [Crawley-Boevey 1991] yield the following useful lemma.

Lemma 3.2. Let $k$ be a field and $S$ a finite poset with a unique maximal element. If an object $M=\left(M_{s} ; s \in S\right)$ of $\operatorname{Fpr}(S, k)$ is a generic representation of $S$, then:

(a) the k-dimension of $M_{\infty}$ is countably infinite;

(b) the $k$-algebra $D_{M}=$ End $M / J($ End $M)$ is a division algebra with $J($ End $M)$ nilpotent; and

(c) the representation $M$ is an object of $\operatorname{fpr}\left(S, D_{M}\right)$ and the $D_{M}$-dimension of $M_{\infty}$ equals the endolength of $M$.

Example 3.3. Assume that $T$ is any of the hypercritical posets $\mathcal{N}_{1}, \ldots, \mathcal{N}_{6}$ of Nazarova and let $T^{\infty}=T \cup\{\infty\}$. In the proof of Theorem 2.4, we have constructed a fully faithful $k$-linear exact functor $F_{T}^{\prime}$ : modf $k\left\langle t_{1}, t_{2}\right\rangle \longrightarrow \operatorname{fpr}\left(T^{\infty}, k\left\langle t_{1}, t_{2}\right\rangle\right)$. We recall from the construction that the modules in $\operatorname{Mod} k\left\langle t_{1}, t_{2}\right\rangle$ are identified with $k$-linear representations of the two-loop quiver

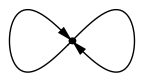

consisting of a single point and two loops $a$ and $b$. Any such representation is a triple $\mathbb{V}=(V, \tilde{a}, \tilde{b})$, where $V$ is a $k$-vector space and $\tilde{a}, \tilde{b}: V \rightarrow V$ are $k$-linear endomorphisms of $V$.

For $j=1, \ldots, 6$, the functor $F_{\mathcal{N}_{j}}^{\prime}$ associates to the representation $\mathbb{V}=(V, \tilde{a}, \tilde{b})$ the object

$$
U_{\mathbb{V}}^{(j)}=F_{\mathcal{N}_{j}}^{\prime}(\mathbb{V})
$$

of the category $\operatorname{Fpr}\left(\mathcal{N}_{j}^{\infty}, k\left\langle t_{1}, t_{2}\right\rangle\right)$ defined by the corresponding diagram $U_{\mathbb{V}}^{(j)}$ from the proof of Theorem 2.4.

Note that the module $k\left\langle t_{1}, t_{2}\right\rangle$ in $\operatorname{Mod} k\left\langle t_{1}, t_{2}\right\rangle$ is identified with the triple $\mathbb{V}=$ $\left(k\left\langle t_{1}, t_{2}\right\rangle, \tilde{t}_{1}, \tilde{t}_{2}\right)$, where $\tilde{t}_{1}, \tilde{t}_{2}$ are the $k$-linear endomorphisms of $k\left\langle t_{1}, t_{2}\right\rangle$ defined by the multiplication by $t_{1}$ and $t_{2}$, respectively. Given $T=\mathcal{N}_{j}$, we define the object 
$U_{k\left\langle t_{1}, t_{2}\right\rangle}^{T}$ of $\operatorname{fpr}\left(T^{\infty}, k\left\langle t_{1}, t_{2}\right\rangle\right)$ to be the object $U_{\mathbb{V}}^{(j)}$, with $\mathbb{V}=\left(k\left\langle t_{1}, t_{2}\right\rangle, \tilde{t}_{1}, \tilde{t}_{2}\right)$. For instance, if $T=\mathcal{N}_{1}$, then $U_{k\left\langle t_{1}, t_{2}\right\rangle}^{T}$ is the object of $\operatorname{fpr}\left(T^{\infty}, k\left\langle t_{1}, t_{2}\right\rangle\right)$ defined by the diagram of $k\left\langle t_{1}, t_{2}\right\rangle$-modules

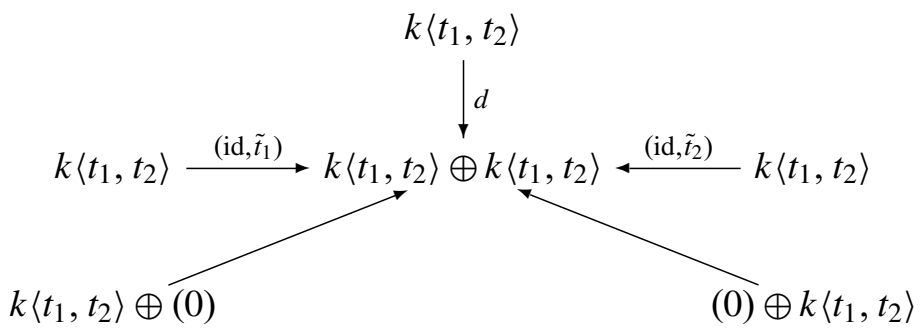

where $d$ is the diagonal embedding, given by $d(x)=(x, x)$, for $x \in k\left\langle t_{1}, t_{2}\right\rangle$.

For each hypercritical poset $T$, clearly $U_{k\left\langle t_{1}, t_{2}\right\rangle}^{T}$ is an object of $\operatorname{fpr}\left(T^{\infty}, k\left\langle t_{1}, t_{2}\right\rangle\right)$ such that $\left(U_{k\left\langle t_{1}, t_{2}\right\rangle}^{T}\right)_{\infty}$ has infinite $k$-dimension and finite $k\left\langle t_{1}, t_{2}\right\rangle$-rank. Since the $k$-linear functor $F_{T}^{\prime}$ is fully faithful, then there are $k$-algebra isomorphisms

$$
k\left\langle t_{1}, t_{2}\right\rangle \cong \operatorname{End}_{k\left\langle t_{1}, t_{2}\right\rangle} k\left\langle t_{1}, t_{2}\right\rangle \cong \operatorname{End} F_{T}^{\prime}\left(k\left\langle t_{1}, t_{2}\right\rangle\right) \cong \operatorname{End} U_{k\left\langle t_{1}, t_{2}\right\rangle}^{T} .
$$

This shows that $U_{k\left\langle t_{1}, t_{2}\right\rangle}^{T}$ is an indecomposable object of $\operatorname{fpr}\left(T^{\infty}, k\left\langle t_{1}, t_{2}\right\rangle\right)$ of finite endolength. Therefore $U_{k\left\langle t_{1}, t_{2}\right\rangle}^{T}$ is a generic object of $\operatorname{Fpr}\left(T^{\infty}, k\left\langle t_{1}, t_{2}\right\rangle\right)$.

The main theorem of this section (Theorem 3.8) gives several characterizations of tame and $k$-wild representation type of the category $\operatorname{fpr}(S, k)$ in terms of existence conditions on generic representations. This theorem parallels that of the main theorem in [Crawley-Boevey 1991] for $\bmod A$ for a finite dimensional algebra $A$ over an algebraically closed field $k$. The proof is different, however, because of the assumption that $k$ is an arbitrary field.

In particular, condition (c) of Theorem 3.8 is an existence condition on generic representations $M$ with End $M / J($ End $M) \cong k(x)$. It is proved in [Crawley-Boevey 1991], using properties of minimal bocses, that if $A$ is a finite-dimensional algebra over an algebraically closed field $k$ and $\bmod A$ has tame representation type, then

$$
\text { End } M / J(\text { End } M) \cong k(x)
$$

for each generic $A$-module $M$. Since the theory of minimal bocses is not available in our context, this condition is included as an assumption in condition (c). The question of whether it is necessary remains unresolved.

The remainder of the paper is devoted to a proof of Theorem 3.8. We start with the following result, proved in [Arnold 2000, Theorem 1.5.3].

Theorem 3.4. Let $k$ be a field and $S$ a finite poset with a unique maximal element. Then $\operatorname{fpr}(S, k)$ has finite representation type if and only if there are no generic representations in $\operatorname{Fpr}(S, k)$. 
We recall from [Arnold and Simson 2002] that the category $\operatorname{fpr}(S, k)$ has tame representation type if for each vector $w=\left(w_{s}\right)_{s \in S} \in \mathbb{Z}^{S}$, with each $w_{s}$ a nonnegative integer, there are finitely many indecomposable objects $N_{1}, \ldots, N_{m_{w}}$ of $\operatorname{fpr}(S, k[x])$ such that if $U$ is an indecomposable object of $\operatorname{fpr}(S, k)$ with $\operatorname{cdn} U=$ $w$, then $U \cong Z \otimes_{k[x]} N_{j}$, for some $1 \leq j \leq m_{w}$ and a cyclic $k[x]$-module $Z$.

Let $N$ be an object of $\operatorname{fpr}(S, k[x])$ and define

$$
\widehat{N}^{x}=k(x) \otimes_{k[x]} N,
$$

an object of $\operatorname{fpr}(S, k(x))$ containing $N$. It follows that the natural embedding $N \hookrightarrow$ $\widehat{N}^{x}$ induces a $k$-algebra embedding End $N \subseteq$ End $\widehat{N}^{x}$. We define the nil radical of End $N$ to be the nilpotent ideal Nil(End $N)=$ End $N \cap J\left(\right.$ End $\left.\widehat{N}^{x}\right)$ of End $N$.

The first step in the proof of Theorem 3.8 is to relate generic representations to certain objects of $\operatorname{fpr}(S, k[x])$. We call an object $N$ of $\operatorname{fpr}(S, k[x])$ pregeneric if $N$ is indecomposable as an object of $\operatorname{Fpr}(S, k)$. Notice that a pregeneric object of $\operatorname{fpr}(S, k[x])$ is also an indecomposable object of $\operatorname{fpr}(S, k[x])$. The terminology, pregeneric representation, is motivated by the following lemma.

Lemma 3.5. Let $k$ be a field and $S$ a finite poset with a unique maximal element.

(a) If $N=\left(N_{s} ; s \in S\right)$ is a pregeneric object of the category $\operatorname{fpr}(S, k[x])$ such that End $N / \operatorname{Nil}($ End $N) \cong k[x]$, then $\widehat{N}^{x}=k(x) \otimes_{k[x]} N$ is a generic object in $\operatorname{Fpr}(S, k)$, the endolength of $\widehat{N}^{x}$ equals the $k[x]$-rank of $N_{\infty}$ and

$$
\text { End } \widehat{N}^{x} / J\left(\text { End } \widehat{N}^{x}\right) \cong k(x) \text {. }
$$

(b) If $M=\left(M_{s} ; s \in S\right)$ is a generic object of $\operatorname{Fpr}(S, k)$ such that

$$
\text { End } M / J(\text { End } M) \cong k(x),
$$

there is a pregeneric object $N=\left(N_{s} ; s \in S\right)$ of $\operatorname{fpr}(S, k[x])$ such that $M \cong \widehat{N}^{x}$, End $N / \operatorname{Nil}($ End $N) \cong k[x]$, and the endolength of $M$ equals the $k[x]$-rank of the free module $N_{\infty}$.

Proof. (a) Clearly, $\widehat{N}^{x}=k(x) \otimes_{k[x]} N$ is an object of $\operatorname{fpr}(S, k(x))$, and hence an object of $\operatorname{Fpr}(S, k)$ with infinite $k$-dimension and finite endolength, since $k(x) \subseteq$ End $\widehat{N}^{x}$. Obviously, there is a $k$-algebra embedding

$$
\text { End } N / \operatorname{Nil}(\text { End } N) \longrightarrow \text { End } \widehat{N}^{x} / J\left(\text { End } \widehat{N}^{x}\right) .
$$

Because $k(x)$ is the quotient field of $k[x] \subseteq$ End $N, N$ is finitely generated and free as a $k[x]$-module, and End $N / \operatorname{Nil}($ End $N) \cong k[x]$, then End $\widehat{N}^{x} / J\left(\right.$ End $\left.\widehat{N}^{x}\right) \cong$ $k(x)$. This shows that $M$ is an indecomposable object of $\operatorname{Fpr}(S, k)$.

(b) By Lemma 3.2, $M_{\infty}$ is a $k(x)$-module, the endolength of the object $M$ equals $\operatorname{dim}_{k(x)} M_{\infty}$ and $M$ is an object of $\operatorname{fpr}(S, k(x))$. Consequently, $M \cong k(x) \otimes_{k[x]} N$ 
for some object $N$ of $\operatorname{fpr}(S, k[x])$ such that the endolength of $M$ equals the $k[x]$ rank of $N_{\infty}$. Since there is an obvious $k$-algebra embedding

$$
\text { End } N / \operatorname{Nil}(\text { End } N) \longrightarrow \text { End } M / J(\text { End } M) \cong k(x),
$$

then the algebra End $N$ has no nontrivial idempotents and therefore $N$ is an indecomposable object of $\operatorname{Fpr}(S, k)$. It follows that End $N / \operatorname{Nil}(\operatorname{End} N) \cong k[x]$.

The next lemma is a characterization of tame representation type in terms of pregeneric representations. The general framework of the proof of the lemma is like that of the proof of Theorem 2.4, in that Case 2 is an induction proof beginning with Case 1 and Case 3 is proved by using properties of representations of clans. However, in the proof of the following lemma, the goal is to construct pregeneric representations in $\operatorname{fpr}(S, k[x])$ rather than finding endomorphism rings of indecomposable objects of $\operatorname{fpr}(S, k)$. We write "almost all" for "finitely many exceptions, up to isomorphism".

Lemma 3.6. Let $k$ be a field and $S$ a finite poset with a unique maximal element. The category $\operatorname{fpr}(S, k)$ has tame representation type if and only if for each vector $w=\left(w_{s}\right)_{s \in S} \in \mathbb{Z}^{S}$, with each $w_{s}$ a nonnegative integer, there are finitely many pregeneric objects $N_{1}, \ldots, N_{m_{w}}$ of $\operatorname{fpr}(S, k[x])$, with End $N_{i} / \operatorname{Nil}\left(\right.$ End $\left.N_{i}\right) \cong k[x]$ for each $i$, such that for almost all indecomposable objects $U$ of $\operatorname{fpr}(S, k)$ with cdn $U=w$, there is an isomorphism $U \cong Z \otimes_{k[x]} N_{j}$, for some $1 \leq j \leq m_{w}$ and $a$ cyclic $k[x]$-module $Z$.

Proof. It is sufficient to assume that $\operatorname{fpr}(S, k)$ has tame representation type and confirm the conditions of the lemma (the converse is clear). The proof follows that of Theorem 2.4.

Case 1. $S=(\cdots) \cup\{\infty\}$.

Define an object $N$ of $\operatorname{fpr}(S, k[x])$ by the formula

$$
N=(k[x] \oplus(0),(0) \oplus k[x], \quad(1,1) k[x], \quad(1, x) k[x], k[x] \oplus k[x]) .
$$

A simple calculation shows that End $N \cong k[x]$, and the endomorphism $k$-algebra of $N$, viewed as an object of $\operatorname{fpr}(S, k)$, is also isomorphic to $k[x]$. It follows that $N$ is a pregeneric representation in $\operatorname{fpr}(S, k[x])$.

It follows from the classification given in [Brenner 1974b] (see also [Simson 1992, Section 15.6]) that, for each $w=\left(w_{s}\right)_{s \in S} \in \mathbb{Z}^{S}$, either

(i) there are only finitely many isomorphism classes of indecomposable objects $U$ of $\operatorname{fpr}(S, k)$ with $\operatorname{cdn} U=w$, or

(ii) each indecomposable object $U$ of $\operatorname{fpr}(S, k)$ is isomorphic to $Z \otimes_{k[x]} N$, for some cyclic $k[x]$-module $Z$. 
Case 2. The poset $S$ does not contain, as a subposet, any of the hypercritical posets $\mathcal{N}_{1}, \mathcal{N}_{2}, \mathcal{N}_{3}, \mathcal{N}_{4}, \mathcal{N}_{5}, \mathcal{N}_{6}$ of Nazarova, or

$$
\mathcal{N} \mathscr{E}=(\stackrel{i \times i}{\cdot} \cdot \cdot) \text {. }
$$

This case is proved by an induction on $p(U)=(|\operatorname{cdn} U|, v(U)) \in \mathbb{N} \times \mathbb{N}^{o p}$, as in [Simson 1992, proof of Theorem 15.54], beginning with Case 1. As summarized in Case II of the proof of Theorem 2.4, if $U$ is an indecomposable sincere object of $\operatorname{fpr}(S, k)$, then either $p\left(U^{\prime}\right)<p(U)$, for $U^{\prime}=\delta_{(a, b)}(U), \operatorname{cdn} U^{\prime}=\operatorname{cdn} U$, and $\delta_{(a, b)} S$ does not contain $\mathcal{N} \mathscr{E}$ as a subposet; or else $U_{a} \subseteq U_{b}$ and $U=u\left(U^{\prime}\right)$, for some object $U^{\prime}$ of $\operatorname{fpr}\left(S_{a<b}, k\right)$ with $p\left(U^{\prime}\right)<p(U)$ and $\operatorname{cdn} U^{\prime}=\operatorname{cdn} U$. In either case, by induction on $p(U)$ beginning with Case 1 , there are finitely many pregeneric objects $N_{1}, \ldots, N_{m_{w}}$ of $\operatorname{fpr}(S, k[x])$ with End $N_{i} / \operatorname{Nil}\left(\right.$ End $\left.N_{i}\right) \cong k[x]$ for each $i$ such that for almost all indecomposable objects $U$ of $\operatorname{fpr}(S, k)$ with $\operatorname{cdn} U=w$, $U \cong Z \otimes_{k[x]} N_{j}$ for some $1 \leq j \leq m_{w}$ and cyclic $k[x]$-module $Z$.

Case 3. The poset $S$ does not contain, as a subposet, any of the posets $\mathcal{N}_{1}, \mathcal{N}_{2}, \mathcal{N}_{3}$, $\mathcal{N}_{4}, \mathcal{N}_{5}$, or $\mathcal{N}_{6}$, but does contain $\mathcal{N}^{\prime} \mathscr{L}$.

This case reduces to the category $\operatorname{rep}_{k}\left(\mathscr{C}_{m, n}\right)$ of $k$-linear representations of a clan $\mathscr{C}_{m, n}$, as outlined in Case III of the proof of Theorem 2.4. There we classified the indecomposable representations in $\operatorname{rep}_{k}\left(\mathscr{C}_{m, n}\right)$ explicitly, into possibilities (a)-(d). (See page 8.)

In (a)-(c), each indecomposable object $U$ of $\operatorname{rep}_{k}\left(\mathscr{C}_{m, n}\right)$ is isomorphic to $S_{w}(M)$, for some cyclic $A_{w}$-module $M$, where $A_{w}$ is a quotient algebra of $k[x]$ and

$$
\text { End } U / J(\text { End } U) \cong \text { End } M / J(\text { End } M) \cong k[x] /(g(x))
$$

is a finite-dimensional field extension of $k$. There is a natural extension of the correspondences

$$
\operatorname{fpr}(S, k) \longrightarrow \operatorname{fpr}\left(\mathscr{G}_{m, n}^{\infty}, k\right) \longrightarrow \operatorname{rep}_{k}\left(\mathscr{C}_{m, n}\right)
$$

to correspondences $\operatorname{Fpr}(S, k) \longrightarrow \operatorname{Fpr}\left(\mathscr{G}_{m, n}^{\infty}, k\right) \longrightarrow \operatorname{Rep}_{k}\left(\mathscr{C}_{m, n}\right)$, where $\operatorname{Rep}_{k}\left(\mathscr{C}_{m, n}\right)$ is the category of all $k$-linear representations of the clan $\mathscr{C}_{m, n}$ (including also infinite dimensional representations). For (a), (b), and (c), $S_{w}(k[x])$ is an indecomposable object of $\operatorname{Rep}_{k}\left(\mathscr{C}_{m, n}\right)$. Let $N$ be the preimage of $S_{w}(k[x])$ in $\operatorname{Fpr}(S, k)$, an indecomposable object in $\operatorname{fpr}(S, k[x])$ with End $N / \operatorname{Nil}($ End $N) \cong k[x]$. Since each indecomposable object $U$ of $\operatorname{rep}_{k}\left(\mathscr{C}_{m, n}\right)$ is isomorphic to $S_{w}(M)$ for some cyclic $A_{w}$-module $M$ of $\operatorname{fpr}(S, k)$ and $A_{w}$ is a factor $k$-algebra of $k[x]$, it follows that if $V$ is an indecomposable object of $\operatorname{fpr}(S, k)$, then $U$ is isomorphic to $Z \otimes_{k[x]} N$ for some cyclic $k[x]$-module $Z$. Thus (d) reduces to Case I. In any case, the conditions of the lemma are satisfied. 
Lemma 3.7. Let $S$ be a finite poset with a unique maximal element, $k$ a field, $N$ a pregeneric object of $\operatorname{fpr}(S, k[x])$ with End $N / \operatorname{Nil}($ End $N) \cong k[x]$ and $d_{N}$ the $k[x]-$ rank of the free $k[x]$-module $N_{\infty}$. Assume that $\operatorname{fpr}(S, k)$ has tame representation type.

(a) There is a torsion cyclic $k[x]$-module $Z_{N}$ such that $U^{N}=Z_{N} \otimes_{k[x]} N=$ $\left(U_{s}^{N} ; s \in S\right)$ is an indecomposable object of $\operatorname{fpr}(S, k)$ and the $k[x]$-rank of $U_{\infty}^{N}$ is equal to $d_{N}$.

(b) Assume that $N^{\prime}$ is another pregeneric object of the category $\operatorname{fpr}(S, k[x])$ such that End $N^{\prime} / \operatorname{Nil}\left(\right.$ End $\left.N^{\prime}\right) \cong k[x]$ and $Z$ is a torsion cyclic $k[x]$-module such that there are $k[x]$-module embeddings $Z_{N} \subseteq Z$ and $Z_{N^{\prime}} \subseteq Z$. Then

\section{$Z_{N} \otimes_{k[x]} N \quad$ and $\quad Z_{N^{\prime}} \otimes_{k[x]} N^{\prime}$}

are isomorphic objects of $\operatorname{fpr}(S, k)$ if and only if $N$ and $N^{\prime}$ are isomorphic objects of $\operatorname{fpr}(S, k[x])$.

Proof. (a) Write $N=\left(N_{s} ; s \in S\right)$. Pick a $k[x]$-basis $B_{s}=\left\{b_{s, i}\right\}$ of $N_{s}$, for each $s \in S$, and express each element $b_{s, i}$ of $B_{s}$ as a $k[x]$-linear combination $b_{s, i}=$ $\sum_{j} f_{s, i, j}(x) b_{\infty, j}$ of elements of $B_{\infty}$. Let $m=\max \left\{\operatorname{deg} f_{s, i, j}(x)\right\}$ and let $Z_{N}=$ $k[x] /\left(x^{m+1}\right)$. We set $U^{N}=Z_{N} \otimes_{k[x]} N$. It is clear that $U^{N}=\left(U_{s}^{N} ; s \in S\right)$ is an object of $\operatorname{fpr}(S, k)$, because $N=\left(N_{s} ; s \in S\right)$ is an object of $\operatorname{fpr}(S, k[x])$ and $U_{s}^{N}=\left(N_{s}+x^{m+1} N_{\infty}\right) / x^{m+1} N_{\infty}$, for each $s \in S$. Since $N_{\infty}$ is a finitely generated free $k[x]$-module and each $N_{s}$ is a finitely generated free summand of $N_{\infty}$, the choice of $m$ guarantees that any $k[x]$-automorphism of $U_{\infty}^{N}$ lifts to a $k[x]$ automorphism of $N_{\infty}$. Therefore, there is a $k$-algebra isomorphism

$$
\text { End } U^{N} / J\left(\text { End } U^{N}\right) \cong \text { End } N / J(\text { End } N) \text {. }
$$

Let $N^{\vee}$ be the representation $N$ viewed as an object of the category $\operatorname{Fpr}(S, k)$. Then there exists a $k$-algebra isomorphism End $N^{\vee} / J\left(\operatorname{End} N^{\vee}\right) \cong$ End $N / J(\operatorname{End} N)$, because End $N^{\vee} / \operatorname{Nil}\left(\right.$ End $\left.N^{\vee}\right) \cong k[x]$ and $\operatorname{Nil}\left(\right.$ End $\left.N^{\vee}\right) \subseteq J\left(\right.$ End $\left.N^{\vee}\right)$. It now follows that End $U^{N} / J\left(\right.$ End $\left.U^{N}\right) \cong$ End $N^{\vee} / J\left(\right.$ End $\left.N^{\vee}\right)$. Since $N$ is an indecomposable object of $\operatorname{Fpr}(S, k), U^{N}$ is an indecomposable object of $\operatorname{fpr}(S, k)$ and the $k[x]$-rank of $U_{\infty}^{N}$ equals $d_{N}$, because $Z_{N}=k[x] /\left(x^{m+1}\right)$.

(b) Clearly, $Z \otimes_{k[x]} N$ and $Z \otimes_{k[x]} N^{\prime}$ are isomorphic objects of $\operatorname{fpr}(S, k)$ if $N$ and $N^{\prime}$ are isomorphic objects of $\operatorname{fpr}(S, k[x])$. An argument similar to that of (a) shows that an isomorphism $Z \otimes_{k[x]} N \simeq Z \otimes_{k[x]} N^{\prime}$ lifts to an isomorphism $N \simeq N^{\prime}$.

Now we are able to prove the main result of this section containing a characterization of tame and $k$-wild representation type of the category $\operatorname{fpr}(S, k)$.

Theorem 3.8. Let $k$ be a field and $S$ a finite poset with a unique maximal element. The following statements are equivalent. 
(a) The category $\mathrm{fpr}(S, k)$ has tame representation type.

(b) The category $\operatorname{fpr}(S, k)$ does not have $k$-wild representation type.

(c) For each integer $d \in \mathbb{N}$, there are only finitely many isoclasses of generic representations $M$ in the category $\operatorname{Fpr}(S, k)$ with endolength $d$ and

$$
\text { End } M / J(\text { End } M) \cong k(x) \text {. }
$$

(d) For each generic representation $M$ in $\operatorname{Fpr}(S, k)$, the algebra

$$
\text { End } M / J(\text { End } M)
$$

does not contain a $k$-subalgebra isomorphic to $k\left\langle t_{1}, t_{2}\right\rangle$.

Proof. (a) $\Longleftrightarrow$ (b): See, for example, [Arnold and Simson 2002, Theorem 2.1; Zavadskij and Nazarova 1977; Zavadskij 1987; 1991].

(a) $\Rightarrow$ (c): Assume that the category $\operatorname{fpr}(S, k)$ has tame representation type and there exist an integer $d \in \mathbb{N}$ and infinitely many pairwise nonisomorphic generic representations $M_{1}, M_{2}, \ldots, M_{m}, \ldots$ in $\operatorname{Fpr}(S, k)$ with endolength $d$,

\section{End $M_{i} / J\left(\right.$ End $\left.M_{i}\right) \cong k(x)$}

for each $i$, and $M_{i} \cong M_{j}$ if and only if $i=j$. By Lemma 3.2, each $M_{i}$ is a $k(x)$ module such that the endolength of $M_{i}=\left(M_{i, s} ; s \in S\right)$ equals $\operatorname{dim}_{k(x)} M_{i, \infty}$, and equals $d$ as well. In addition,

$$
M_{i} \cong k(x) \otimes_{k[x]} N_{i}
$$

for some pregeneric object $N_{i}=\left(N_{i, s} ; s \in S\right)$ of $\operatorname{fpr}(S, k[x])$ such that the $k[x]$ rank of $N_{i, \infty}$ equals $d$ and End $N_{i} / \operatorname{Nil}\left(\right.$ End $\left.N_{i}\right) \cong k[x]$ (see Lemma 3.5). Moreover, if $N_{i} \cong N_{j}$, then $M_{i} \cong M_{j}$ and so $i=j$. Consequently, there must be infinitely many isomorphism classes of the pregeneric objects $N_{1}, N_{2}, \ldots, N_{m}, \ldots$

If $N=\left(N_{s} ; s \in S\right)$ is a pregeneric object of $\operatorname{fpr}(S, k[x])$ such that

\section{End $N / \operatorname{Nil}($ End $N) \cong k[x]$}

and the $k[x]$-rank of $N_{\infty}$ equals $d$ then, by Lemma 3.5, $\widehat{N}^{x}=k(x) \otimes_{k[x]} N$ is a generic representation in $\operatorname{Fpr}(S, k)$ with endolength $d$ and End $\widehat{N}^{x} / J\left(\right.$ End $\left.\widehat{N}^{x}\right) \cong$ $k(x)$. Hence, $\widehat{N}^{x} \cong M_{i}$ for some $i$. It now follows from Lemma 3.7 that there is some $w=\left(w_{s}\right)_{s \in S}$ with $w_{\infty}=d$ such that, up to isomorphism, infinitely many pregeneric objects $N$ of $\operatorname{fpr}(S, k[x])$ with End $N / \operatorname{Nil}($ End $N) \cong k[x]$ are required so that each indecomposable $U$ of $\operatorname{fpr}(S, k)$, with $w=\operatorname{cdn} U$, is isomorphic to $Z \otimes_{k[x]} N$ for some cyclic $k[x]$-module $Z$. This is a contradiction to Lemma 3.6 and the assumption that $\operatorname{fpr}(S, k)$ has tame representation type. 
(c) $\Rightarrow$ (b): We follow [Crawley-Boevey 1991, Theorem 4.4]. Assume that $\operatorname{fpr}(S, k)$ has $k$-wild representation type. In view of Lemma 3.1, there is some

$$
N \in \operatorname{fpr}\left(S, k\left\langle t_{1}, t_{2}\right\rangle\right)
$$

such that the $k$-linear functor $-\otimes_{k\left\langle t_{1}, t_{2}\right\rangle} N^{\vee}: \operatorname{Mod} k\left\langle t_{1}, t_{2}\right\rangle \longrightarrow \operatorname{Fpr}(S, k)$ is exact, preserves isomorphism classes, preserves indecomposables and restricts to the functor $-\otimes_{k\left\langle t_{1}, t_{2}\right\rangle} N^{\vee}:$ modf $k\left\langle t_{1}, t_{2}\right\rangle \longrightarrow \mathrm{fpr}(S, k)$, where $N^{\vee}$ is the representation $N$ viewed as an object of $\operatorname{Fpr}(S, k)$. For each $\lambda \in k(x)$, we define

$$
M_{\lambda}=(k(x)[y] /(y-\lambda)) \otimes_{k\left\langle t_{1}, t_{2}\right\rangle} N^{\vee},
$$

an indecomposable object of $\operatorname{Fpr}(S, k)$. Then $M_{\lambda}$ has infinite $k$-dimension and $d$ is equal to the $k\left\langle t_{1}, t_{2}\right\rangle$-rank of $N_{\infty}$ as well as to the endolength of $M_{\lambda}$, and this number is finite, for each $\lambda \in k(x)$. Consequently, each $M_{\lambda}$ is a generic representation in $\operatorname{Fpr}(S, k)$. Since End $M_{\lambda} / J\left(\right.$ End $\left.\left.M_{\lambda}\right)\right) \cong k(x)$, for each $\lambda$, and $M_{\lambda} \cong M_{\sigma}$ if and only if $\lambda=\sigma$, the proof is complete.

(d) $\Rightarrow$ (b): Assume that $\operatorname{fpr}(S, k)$ has $k$-wild representation type. Then $S$ contains a subposet $T$ isomorphic to one of the 6 hypercritical posets $\mathcal{N}_{1}, \mathcal{N}_{2}, \mathcal{N}_{3}, \mathcal{N}_{4}, \mathcal{N}_{5}$, or $\mathcal{N}_{6}$ of Nazarova [Nazarova 1975], see also [Simson 1992, Theorem 15.3]. As noted in Example 3.3, for each $T$, there is an object $U_{k\left\langle t_{1}, t_{2}\right\rangle}^{T}$ of $\operatorname{fpr}\left(T^{\infty}, k\left\langle t_{1}, t_{2}\right\rangle\right)$ such that End $U_{k\left\langle t_{1}, t_{2}\right\rangle}^{T} \cong k\left\langle t_{1}, t_{2}\right\rangle$. It was shown in the proof of Theorem 2.4 that there exists a fully faithful $k$-linear functor

$$
H_{T}: \mathrm{fpr}\left(T^{\infty}, k\left\langle t_{1}, t_{2}\right\rangle\right) \longrightarrow \mathrm{fspr}\left(S, k\left\langle t_{1}, t_{2}\right\rangle\right) .
$$

It follows that $N_{T}=H_{T}\left(U_{k\left\langle t_{1}, t_{2}\right\rangle}^{T}\right)$ is an object of $\operatorname{fspr}\left(S, k\left\langle t_{1}, t_{2}\right\rangle\right)$ such that

$$
\text { End } N_{T} \cong \text { End } N_{T}^{\vee} \cong \text { End } U_{k\left\langle t_{1}, t_{2}\right\rangle}^{T} \cong k\left\langle t_{1}, t_{2}\right\rangle \text {. }
$$

It follows from [Kasjan 2003, Corollary 6.1] that there exists such fully faithful $k$-linear functor $H_{T}$ that satisfies the following three conditions:

- $N_{T}=H_{T}\left(U_{k\left\langle t_{1}, t_{2}\right\rangle}^{T}\right)$ is an object of $\operatorname{fpr}\left(S, k\left\langle t_{1}, t_{2}\right\rangle\right)$,

- there are $k$-algebra isomorphisms End $N_{T} \cong$ End $N_{T}^{\vee} \cong$ End $U_{k\left\langle t_{1}, t_{2}\right\rangle}^{T} \cong k\left\langle t_{1}, t_{2}\right\rangle$,

- the $k$-linear functor $-\otimes_{k\left\langle t_{1}, t_{2}\right\rangle} N_{k S}: \operatorname{Mod} k\left\langle t_{1}, t_{2}\right\rangle \longrightarrow \operatorname{Fpr}(S, k)$ is full, faithful and exact.

Let $D$ be the universal division algebra of fractions of $k\left\langle t_{1}, t_{2}\right\rangle$. Then the representation $\widehat{N}_{T}^{D}=D \otimes_{k\left\langle t_{1}, t_{2}\right\rangle} N_{T}$ is an indecomposable object in $\operatorname{Fpr}(S, k)$ such that

$$
\text { End } \widehat{N}_{T}^{D} \cong D \otimes_{k\left\langle t_{1}, t_{2}\right\rangle} \text { End } N_{T} \cong D \otimes_{k\left\langle t_{1}, t_{2}\right\rangle} k\left\langle t_{1}, t_{2}\right\rangle \cong D \text {. }
$$

Moreover, the representation $\widehat{N}_{T}^{D}=\left(\left(\widehat{N}_{T}^{D}\right)_{s} ; s \in S\right)$ has infinite $k$-dimension and finite endolength, since the endolength of $\widehat{N}_{T}^{D}$ is equal to the $D$-dimension of 
$\left(\widehat{N}_{T}^{D}\right)_{\infty}$, as well as to the $k\left\langle t_{1}, t_{2}\right\rangle$-rank of $\left(N_{T}\right)_{\infty}$. Therefore $\widehat{N}_{T}^{D}$ is a generic representation in $\operatorname{Fpr}(S, k)$ and End $\widehat{N}_{T}^{D}$ contains a subalgebra isomorphic to $k\left\langle t_{1}, t_{2}\right\rangle$.

(a) $\Rightarrow$ (d): Let $M$ be a generic representation in the category $\operatorname{Fpr}(S, k)$ such that $d$ is the endolength of $M$ and the $k$-algebra End $M / J($ End $M)$ contains as a subalgebra a universal division $k$-algebra $D$ of fractions of $k\left\langle t_{1}, t_{2}\right\rangle$. Then $M \supseteq$ $D \otimes_{k\left\langle t_{1}, t_{2}\right\rangle} N$ for some indecomposable object $N=\left(N_{s} ; s \in S\right)$ of $\operatorname{fpr}\left(S, k\left\langle t_{1}, t_{2}\right\rangle\right)$ such that the $k\left\langle t_{1}, t_{2}\right\rangle$-rank of $N_{\infty}$ equals $d$ and there is a $k$-algebra isomorphism End $N / J($ End $N) \cong k\left\langle t_{1}, t_{2}\right\rangle$. Given irreducible polynomials $f\left(t_{1}\right) \in k\left[t_{1}\right]$ and $g\left(t_{2}\right) \in k\left[t_{2}\right]$ and positive integers $i$ and $j$, we consider the quotient $k$-algebra

$$
R=k\left\langle t_{1}, t_{2}\right\rangle /\left(t_{1} t_{2}, t_{2} t_{1}, f\left(t_{1}\right)^{i}, g\left(t_{2}\right)^{j}\right)
$$

of $k\left\langle t_{1}, t_{2}\right\rangle$ and the indecomposable object $U=R \otimes_{k\left\langle t_{1}, t_{2}\right\rangle} N$ of $\operatorname{fpr}(S, k)$ with endomorphism ring $R$. Then $\operatorname{fpr}(S, k)$ has $k$-endo-wild representation type, because each finite-dimensional $k$-algebra $A$ is isomorphic to the centralizer of two matrices $M$ and $N$ with minimal polynomials $f\left(t_{1}\right)^{i}$ and $g\left(t_{2}\right)^{j}$, respectively, see [Brenner 1974a]. Therefore, there are $k$-algebra isomorphisms $A \cong R \cong$ End $U$ and, according to Theorem 2.4, the category $\operatorname{fpr}(S, k)$ has $k$-wild representation type.

The following corollary shows that if $\operatorname{fpr}(S, k)$ has tame representation type, then (finite-dimensional) indecomposable objects of $\operatorname{fpr}(S, k)$ can be constructed from (infinite-dimensional) generic representations in $\operatorname{Fpr}(S, k)$.

Corollary 3.9. Let $k$ be a field and $S$ a finite poset with a unique maximal element. If $\operatorname{fpr}(S, k)$ has tame representation type, then all indecomposable objects $U$ of $\operatorname{fpr}(S, k)$ can be constructed as follows.

(a) Choose a generic object $M$ in $\operatorname{Fpr}(S, k)$ with End $M / J($ End $M) \cong k(x)$.

(b) Write $M=k(x) \otimes_{k[x]} N$, where $N$ is some pregeneric object of the category $\operatorname{fpr}(S, k[x])$ with End $N / J($ End $N) \cong k[x]$.

(c) There is an object $U$ of $\operatorname{fpr}(S, k)$ such that $U \cong Z \otimes_{k[x]} N$, for some cyclic $k[x]$-module $Z$.

Proof. Apply Lemmas 3.6 and 3.7.

\section{Appendix}

In the proof of Theorem 2.4, we essentially used the following theorem proved in [Simson 1992, pp. 311-312]. Since there are some inaccuracies in the proof given in [Simson 1992], we present here, for convenience of the reader, a correct construction of the functors $\widehat{F}_{\mathcal{N}_{1}}, \ldots, \widehat{F}_{\mathcal{N}_{6}}$. 
Theorem A.1. If $\mathcal{N}$ is any of the hypercritical posets $\mathcal{N}_{1}, \ldots, \mathcal{N}_{6}$ of Nazarova presented in Section 2, then there exists a fully faithful exact $k$-linear functor $\widehat{F}_{\mathcal{N}}$ : $\operatorname{Mod} k\left\langle t_{1}, t_{2}\right\rangle \longrightarrow \operatorname{Fpr}\left(\mathcal{N}^{\infty}, k\right)$, which restricts to the functor

$$
\widehat{F}_{\mathcal{N}}: \operatorname{modf} k\left\langle t_{1}, t_{2}\right\rangle \longrightarrow \operatorname{fpr}\left(\mathcal{N}^{\infty}, k\right) \cap \operatorname{prin} k \mathcal{N}^{\infty} .
$$

Proof. We follow the proof given in [Simson 1992, pp. 310-312]. We recall that $\operatorname{fpr}\left(\mathcal{N}^{\infty}, k\right)$ is the category $\mathcal{N}$-sp of $\mathcal{N}$-spaces over $k$ studied in [Simson 1992].

We define a functor $\widehat{F}_{\mathcal{N}}: \operatorname{Mod} k\left\langle t_{1}, t_{2}\right\rangle \longrightarrow \operatorname{Fpr}\left(\mathcal{N}^{\infty}, k\right)$, for any $\mathcal{N} \in\left\{\mathcal{N}_{1}, \ldots, \mathcal{N}_{6}\right\}$. For this purpose, we recall from [Simson 1992, Section 14.1] that the modules in $\operatorname{Mod} k\left\langle t_{1}, t_{2}\right\rangle$ are identified with $k$-linear representations of the two-loop quiver

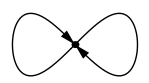

consisting of a single point and two loops $a$ and $b$. Any such representation is a triple $\mathbb{V}=(V, \tilde{a}, \tilde{b})$, where $V$ is a $k$-vector space and $\tilde{a}, \tilde{b}: V \rightarrow V$ are $k$ linear endomorphisms of $V$. For $j=1, \ldots, 6$, the functor $\widehat{F}_{\mathcal{N}_{j}}$ associates to the representation $\mathbb{V}=(V, \tilde{a}, \tilde{b})$ the object $U_{\mathbb{V}}^{(j)}=\widehat{F}_{\mathcal{N}_{j}}(\mathbb{V})$ of the category $\operatorname{Fpr}\left(\mathcal{N}_{j}^{\infty}, k\right)$ defined by the following diagrams, where $O$ is the zero vector space and we set $O^{i} \oplus V^{j}=\underbrace{O \oplus \cdots \oplus O}_{i} \oplus \underbrace{V \oplus \cdots \oplus V}_{j}$

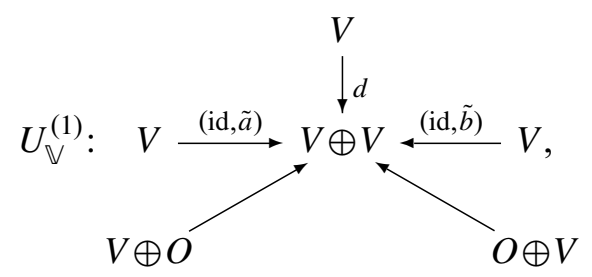

where $d(x)=(x, x)$;

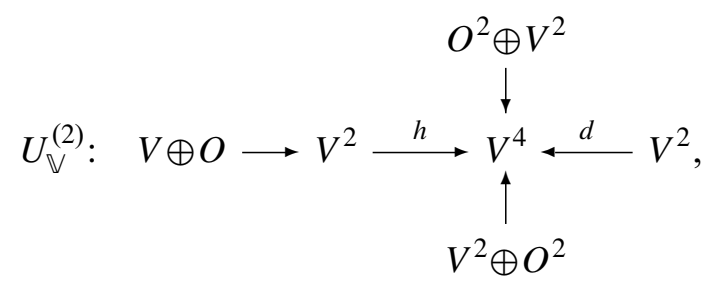

where $d(x, y)=(x, y, x, y)$ and $h(x, y)=(x, y, \tilde{a}(x)+\tilde{b}(y), x)$;

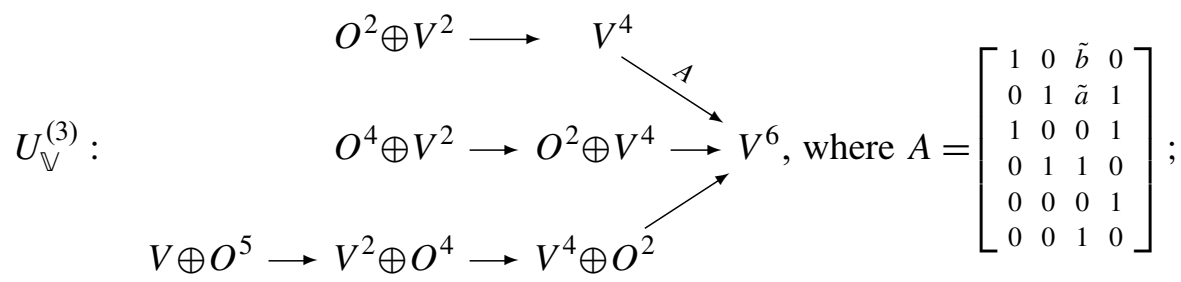




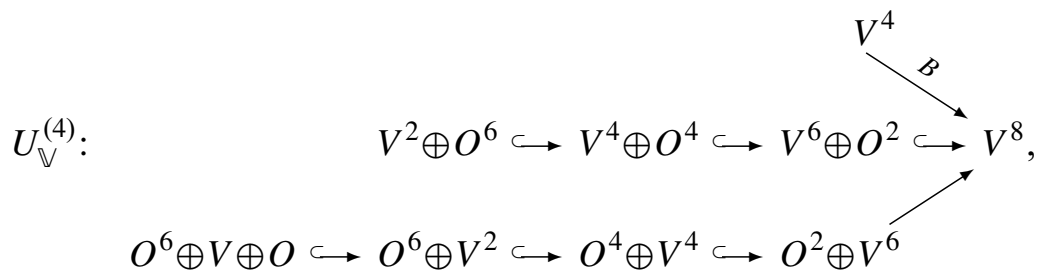

where $B=\left[\begin{array}{cccc}0 & 1 & 1 & 0 \\ \tilde{a} & \tilde{b} & 0 & 1 \\ 1 & 0 & 1 & 0 \\ 0 & 1 & 0 & 1 \\ 0 & 0 & 1 & 0 \\ 0 & 0 & 0 & 1 \\ 1 & 0 & 0 & 0 \\ 0 & 1 & 0 & 0\end{array}\right]$;

$U_{\mathbb{V}}^{(5)}:$

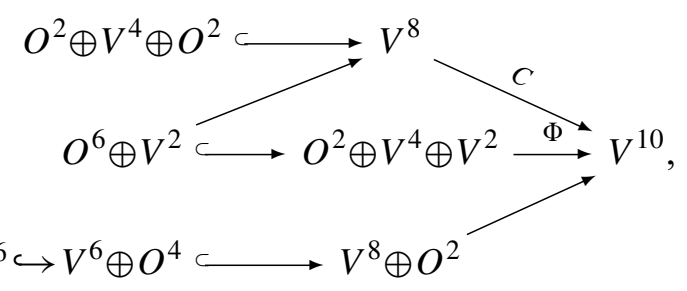

$O \oplus V \oplus O^{8} \hookrightarrow V^{2} \oplus O^{8} \hookrightarrow V^{4} \oplus O^{6} \hookrightarrow V^{6} \oplus O^{4} \hookrightarrow V^{8} \oplus O^{2}$

where $C=\left[\begin{array}{cccccccc}0 & 0 & 0 & 0 & 0 & 0 & 0 & 0 \\ 0 & 0 & 0 & 0 & 0 & 0 & 0 & 0 \\ 1 & 0 & 0 & 0 & 0 & 0 & 0 & 0 \\ 0 & 1 & 0 & 0 & 0 & 0 & 0 & 0 \\ 0 & 0 & 1 & 0 & 0 & 0 & 0 & 0 \\ 0 & 0 & 0 & 1 & 0 & 0 & 0 & 0 \\ 0 & 0 & 0 & 0 & 1 & 0 & 0 & 0 \\ 0 & 0 & 0 & 0 & 0 & 1 & 0 & 0 \\ 0 & 0 & 1 & 0 & 0 & 0 & 1 & 0 \\ 0 & 0 & 0 & 1 & 0 & 0 & 0 & 1\end{array}\right]$ and $\Phi=O^{2} \oplus D \oplus \operatorname{id}_{V^{2}}$ with $D=\left[\begin{array}{cccc}0 & 1 & 1 & 0 \\ \tilde{a} & \tilde{b} & 0 & 1 \\ 1 & 0 & 1 & 0 \\ 0 & 1 & 0 & 1 \\ 0 & 0 & 1 & 0 \\ 0 & 0 & 0 & 1 \\ 1 & 0 & 0 & 0 \\ 0 & 1 & 0 & 0 \\ 0 & 0 & 0 & 0 \\ 0 & 0 & 0 & 0\end{array}\right]$;

and finally

$U_{\Downarrow}^{(6)}:$

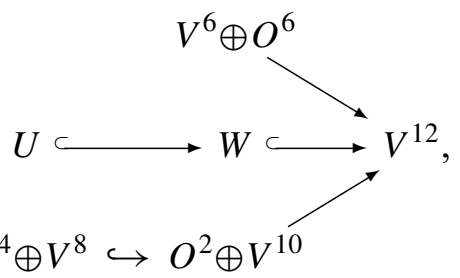

where

$W=\left\{\left(w_{1}, w_{2}, w_{3}, w_{4}, w_{5}, w_{6}, w_{7}, w_{8}, w_{5}, w_{6}, w_{3}, w_{4}\right) ; w_{i} \in V\right.$ for $\left.1 \leq i \leq 8\right\}$,

$U=\left\{w \in W ; w_{5}=w_{1}+w_{3}, w_{6}=w_{2}+\tilde{a}\left(w_{4}\right), w_{7}=w_{2}+w_{3}+\tilde{b}\left(w_{4}\right)\right.$, $\left.w_{8}=w_{1}+w_{4}, w_{9}=w_{1}+w_{3}, w_{10}=w_{2}+\tilde{a}\left(w_{4}\right), w_{11}=w_{3}, w_{12}=w_{4}\right\}$. 
A straightforward calculation shows that, for each $j=1, \ldots, 6$, we have defined a fully faithful exact $k$-linear functor $\widehat{F}_{\mathcal{N}_{j}}: \operatorname{Mod} k\left\langle t_{1}, t_{2}\right\rangle \longrightarrow \operatorname{Fpr}\left(\mathcal{N}_{j}^{\infty}, k\right)$, which restricts to the functor $\widehat{F}_{\mathcal{N}_{j}}$ : modf $k\left\langle t_{1}, t_{2}\right\rangle \longrightarrow \operatorname{fpr}\left(\mathcal{N}_{j}^{\infty}, k\right)=\mathcal{N}_{j}$-sp. The arguments given in [Simson 1992, p. 312] show that $\operatorname{Im} \widehat{F}_{\mathcal{N}_{j}} \subseteq \operatorname{prin} k \mathcal{N}_{j}^{\infty}$, for $j=1, \ldots, 6$. This finishes the proof.

\section{References}

[Arnold 2000] D. M. Arnold, Abelian groups and representations of finite partially ordered sets, CMS Books in Mathematics 2, Springer, New York, 2000. MR 2001g:16030 Zbl 0959.16011

[Arnold and Simson 2002] D. M. Arnold and D. Simson, "Representations of finite partially ordered sets over commutative uniserial rings", preprint, 2002.

[Arnold et al. 1993] D. Arnold, F. Richman, and C. Vinsonhaler, "Representations of finite posets and valuated groups", J. Algebra 155:1 (1993), 110-126. MR 94d:20064 Zbl 0784.20026

[Brenner 1974a] S. Brenner, "Decomposition properties of some small diagrams of modules", pp. 127-141 in Symposia Mathematica (Rome, 1972), vol. 13, Academic Press, London, 1974. MR 50 \#13159 Zbl 0289.16019

[Brenner 1974b] S. Brenner, “On four subspaces of a vector space”, J. Algebra 29 (1974), 587-599. MR 49 \#7271 Zbl 0279.15002

[Butler 1968] M. C. R. Butler, "Torsion-free modules and diagrams of vector spaces", Proc. London Math. Soc. (3) 18 (1968), 635-652. MR 37 \#6327 Zbl 0179.32603

[Corner 1963] A. L. S. Corner, "Every countable reduced torsion-free ring is an endomorphism ring”, Proc. London Math. Soc. (3) 13 (1963), 687-710. MR 27 \#3704 Zbl 0116.02403

[Crawley-Boevey 1988] W. W. Crawley-Boevey, "On tame algebras and bocses", Proc. London Math. Soc. (3) 56:3 (1988), 451-483. MR 89c:16028 Zbl 0661.16026

[Crawley-Boevey 1989] W. W. Crawley-Boevey, “Functorial filtrations, II: Clans and the Gel'fand problem”, J. London Math. Soc. (2) 40:1 (1989), 9-30. MR 91i:16025 Zbl 0725.16012

[Crawley-Boevey 1991] W. Crawley-Boevey, "Tame algebras and generic modules", Proc. London Math. Soc. (3) 63:2 (1991), 241-265. MR 92m:16019 Zbl 0741.16005

[Crawley-Boevey 1992] W. Crawley-Boevey, "Modules of finite length over their endomorphism rings", pp. 127-184 in Representations of algebras and related topics (Kyoto, 1990), edited by H. Tachikawa and S. Brenner, London Math. Soc. Lecture Note Ser. 168, Cambridge Univ. Press, Cambridge, 1992. MR 94h:16018 Zbl 0805.16028

[Deng 2000] B. Deng, “On a problem of Nazarova and Roiter", Comment. Math. Helv. 75:3 (2000), 368-409. MR 2002d:16014 Zbl 0972.16005

[Drozd 1979] J. A. a. Drozd, “Tame and wild matrix problems", pp. 39-74 in Представления и квадратичные формы, edited by J. A. Mitropol'skiı̌, Akad. Nauk Ukrain. SSR Inst. Mat., Kiev, 1979. In Russian; translated in Amer. Math. Soc. Transl. Ser. 2 128, 31-55 (1986. MR 82m:16028 Zbl 0454.16014

[Dugas and Rangaswamy 2002] M. Dugas and K. M. Rangaswamy, "Completely decomposable abelian groups with a distinguished cd subgroup”, Rocky Mountain J. Math. 32:4 (2002), 13831395. MR 2004e:20094 Zbl 1035.20042

[Fuchs 1973] L. Fuchs, Infinite abelian groups, vol. II, Pure and Applied Math. 36, Academic Press, New York, 1973. MR 50 \#2362 Zbl 0257.20035 
[Geiß 1999] C. Geiß, "Maps between representations of clans", J. Algebra 218:1 (1999), 131-164. MR 2000h:16021 Zbl 0978.16010

[Han 2001a] Y. Han, “Controlled wild algebras”, Proc. London Math. Soc. (3) 83:2 (2001), 279298. MR 2002g:16025 Zbl 1019.16007

[Han 2001b] Y. Han, "Strictly wild algebras with radical square zero", Arch. Math. (Basel) 76:2 (2001), 95-99. MR 2002a:16020 Zbl 0980.16010

[Han 2002] Y. Han, "Wild radical square zero algebras", Sci. China Ser. A 45:1 (2002), 29-32. MR 2003b:16015 Zbl 01782157

[Kasjan 2003] S. Kasjan, "On fully wild categories of representations of posets", preprint, Toruń, 2003.

[Kasjan and Simson 1995] S. Kasjan and D. Simson, "Fully wild prinjective type of posets and their quadratic forms”, J. Algebra 172:2 (1995), 506-529. MR 96m:16020 Zbl 0831.16010

[Krause 1991] H. Krause, "Maps between tree and band modules", J. Algebra 137:1 (1991), 186194. MR 92j:16010 Zbl 0715.16007

[Mader 2000] A. Mader, Almost completely decomposable groups, Algebra, Logic and Applications 13, Gordon and Breach, Amsterdam, 2000. MR 2001c:20114 Zbl 0945.20031

[Nazarova 1975] L. A. Nazarova, "Partially ordered sets of infinite type", Izv. Akad. Nauk SSSR Ser. Mat. 39:5 (1975), 963-991, 1219. In Russian. MR 53 \#10664 Zbl 0362.06001

[Nongxa and Vinsonhaler 1996] L. G. Nongxa and C. Vinsonhaler, "Balanced Butler groups", J. Algebra 180:2 (1996), 546-570. MR 97d:20069 Zbl 0846.20060

[Richman and Walker 1999] F. Richman and E. A. Walker, "Subgroups of $p^{5}$-bounded groups", pp. 55-73 in Abelian groups and modules (Dublin, 1998), edited by P. C. Eklof and R. Gobel, Birkhäuser, Basel, 1999. MR 2000m:13035 Zbl 0953.20045

[Ringel 1976] C. M. Ringel, "Representations of $K$-species and bimodules", J. Algebra 41:2 (1976), 269-302. MR 54 \#10340 Zbl 0338.16011

[Ringel 2002] C. M. Ringel, “Combinatorial representation theory: history and future”, pp. 122-144 in Representations of algebras, vol. I, edited by D. Happel and Y. B. Zhang, Beijing Norm. Univ. Press, Beijing, 2002. MR 2067375

[Ringel and Schmidmeier 2004] C. M. Ringel and M. Schmidmeier, "Submodule categories of wild representation type", preprint, 2004. math.RT/0409417

[Schmidmeier 2004] M. Schmidmeier, "Bounded submodules of modules", 2004. math.RT/0408181 [Simson 1992] D. Simson, Linear representations of partially ordered sets and vector space categories, Algebra, Logic and Applications 4, Gordon and Breach, Montreux, 1992. MR 95g:16013 Zbl 0818.16009

[Simson 1993] D. Simson, "On representation types of module subcategories and orders", Bull. Polish Acad. Sci. Math. 41:2 (1993), 77-93 (1994). MR 97g:16024 Zbl 0805.16011

[Simson 2002] D. Simson, "An endomorphism algebra realization problem and Kronecker embeddings for algebras of infinite representation type", J. Pure Appl. Algebra 172:2-3 (2002), 293-303. MR 2003g:16039 Zbl 1017.16019

[Simson 2003] D. Simson, "On large indecomposable modules, endo-wild representation type and right pure semisimple rings”, Algebra Discrete Math. no. 2 (2003), 93-118. MR 2048658

[Zavadskij 1977] A. G. Zavadskiŭ, "Differentiation with respect to a pair of places", pp. 115-121 in Матричные задачи, edited by J. A. Mitropol'skiı̆, Akad. Nauk Ukrain. SSR Inst. Mat., Kiev, 1977. In Russian. MR 58 \#21874a Zbl 0457.06001 
[Zavadskij 1987] A. G. Zavadskiı̌, "A differentiation algorithm and a classification of representations”, Kievskij Ordena Trudovova Krasnovo Znameni Inžinerno-Stroitelnyi Institut (KISI), Dep. Ukr. NIINTI, Kiev, 1987. in Russian.

[Zavadskij 1991] A. G. Zavadskiı̆, "A differentiation algorithm and a classification of poset representations", Izv. Akad. Nauk SSSR Ser. Mat. 55:5 (1991), 1007-1048. In Russian; translation in Math. USSR Izv. 39:2 (1992), 975-1012. MR 93c:16015 Zbl 0809.16011

[Zavadskij and Nazarova 1977] A. G. Zavadskiı̌ and L. A. Nazarova, "Partially ordered sets of tame type”, pp. 122-143 in Матричные задачи, Akad. Nauk Ukrain. SSR Inst. Mat., Kiev, 1977. In Russian. MR 58 \#21874b Zbl 0461.16024

Received May 19, 2003. Revised April 14, 2004.

DAVID M. ARNOLD

DEPARTMENT OF MATHEMATICS

BAYLOR UNIVERSITY

WACO, TX 76798-7328

UNITED STATES

David_Arnold@baylor.edu

DANIEL SIMSON

FACUlty of MATHEMATICS AND COMPUTER SCIENCE

NICOLAUS COPERNICUS UNIVERSITY

UL. CHOPINA $12 / 18$

87-100 TORUŃ

POLAND

simson@mat.uni.torun.pl 